\title{
Optimal Orlicz-Sobolev embeddings
}

Andrea Cianchi

\begin{abstract}
An embedding theorem for the Orlicz-Sobolev space $W_{0}^{1, A}(G)$, $G \subset \mathbb{R}^{n}$, into a space of Orlicz-Lorentz type is established for any given Young function $A$. Such a space is shown to be the best possible among all rearrangement invariant spaces. A version of the theorem for anisotropic spaces is also exhibited. In particular, our results recover and provide a unified framework for various well-known Sobolev type embeddings, including the classical inequalities for the standard Sobolev space $W_{0}^{1, p}(G)$ by O'Neil and by Peetre $(1 \leq p<n)$, and by Brezis-Wainger and by Hansson $(p=n)$.
\end{abstract}

\section{Introduction and main results}

A form of the standard Sobolev inequality, also called Sobolev embedding theorem, amounts to the following statement. Let $G$ be an open subset of $\mathbb{R}^{n}, n \geq 2$, and let $W_{0}^{1, p}(G), 1 \leq p \leq \infty$, denote the first order Sobolev space of those real-valued weakly differentiable functions in $G$, vanishing on $\partial G$, whose gradient belongs to $L^{p}(G)$. If $1 \leq p<n$, then a constant C, depending only on $n$ and $p$ exists such that

$$
\|u\|_{L^{p^{*}}(G)} \leq C\|\mid \nabla u\|_{L^{p}(G)}
$$

for every $u \in W_{0}^{1, p}(G)$. Here

$$
p^{*}=\frac{n p}{n-p}
$$

the Sobolev conjugate of $p$, and $\nabla$ stands for gradient (see e.g. [24, 34]).

2000 Mathematics Subject Classification: 46E35, 46E30.

Keywords: Sobolev inequalities, Orlicz spaces, rearrangement invariant spaces, interpolation. 
If $p>n$ and the Lebesgue measure $|G|$ of $G$ is finite, then there exists a constant $\mathrm{C}$, depending on $n, p$ and $|G|$, such that

$$
\|u\|_{L^{\infty}(G)} \leq C\|\| \nabla u \|_{L^{p}(G)}
$$

for every $u \in W_{0}^{1, p}(G)$.

In the borderline situation where $p=n$, inequality (1.1) is known to hold, for every $G$ having finite measure, with $p^{*}$ replaced by any number greater than or equal to 1 . However, a stronger result, proved in [31] (see also $[27,33])$ is available. Namely,

$$
\|u\|_{\exp L^{n^{\prime}}(G)} \leq C\||\nabla u|\|_{L^{n}(G)}
$$

for some constant $C$, depending only on $n$ and $|G|$, and for every $u \in$ $W_{0}^{1, n}(G)$. Here, $\exp L^{n^{\prime}}(G)$ denotes the Orlicz space associated with the Young function $\exp ^{n^{\prime}}(s)=e^{s^{n^{\prime}}}-1$, and $n^{\prime}=\frac{n}{n-1}$, the Hölder conjugate of $n$. Throughout the paper, a function $A:[0, \infty) \rightarrow[0, \infty]$ will be called a Young function if it is convex (non trivial), left-continuous and vanishes at 0; thus, any Young function $A$ admits the representation

$$
A(s)=\int_{0}^{s} a(r) d r \quad \text { for } s \geq 0
$$

where $a:[0, \infty) \rightarrow[0, \infty]$ is a non-decreasing, left-continuous function, which is neither identically equal to 0 nor to $\infty$. The Orlicz space $L^{A}(G)$ is the Banach space of those real-valued measurable functions $u$ in $G$ for which the Luxemburg norm

$$
\|u\|_{L^{A}(G)}=\inf \left\{\lambda>0: \int_{G} A\left(\frac{|u|}{\lambda}\right) d x \leq 1\right\}
$$

is finite. Note that $L^{A}(G)=L^{p}(G)$ if $A(s)=s^{p}$ for some $p \in[1, \infty)$; moreover, $L^{A}(G)=L^{\infty}(G)$ (up to equivalent norms) if $A(s)$ is any Young function which vanishes for small $s$ and equals $\infty$ for large $s$.

After the contributions of [16] and [1], and the result of [29] dealing with embeddings into $L^{\infty}(G)$, in [10] we established a sharp extension of inequalities (1.1) - (1.3) to the case where Lebesgue norms are replaced by any Orlicz norm on the right-hand side. This result, in an equivalent form contained in [11]-[14], can be stated as follows. Let $G$ be either $\mathbb{R}^{n}$, or any open subset of $\mathbb{R}^{n}$ having finite measure. Given any Young function $A$ such that

$$
\int_{0}\left(\frac{r}{A(r)}\right)^{\frac{1}{n-1}} d r<\infty
$$


define $A_{n}:[0, \infty) \rightarrow[0, \infty]$ as

$$
A_{n}(s)=A \circ H_{n}^{-1}(s) \text { for } s \geq 0,
$$

where $H_{n}{ }^{-1}$ is the (generalized) left-continuous inverse of the function $H_{n}$ : $[0, \infty) \rightarrow[0, \infty)$ given by

$$
H_{n}(r)=\left(\int_{0}^{r}\left(\frac{t}{A(t)}\right)^{\frac{1}{n-1}} d t\right)^{\frac{1}{n^{\prime}}} \text { for } r \geq 0
$$

(and $A(\infty)$ is taken equal to $\infty$ ). Then $A_{n}$ is a Young function and there exists a constant $C$ such that

$$
\|u\|_{L^{A_{n}(G)}} \leq C\||\nabla u|\|_{L^{A}(G)}
$$

for every function $u$ from the Orlicz-Sobolev space $W_{0}^{1, A}(G)$ of those weakly differentiable functions in $G$, which decay to 0 on $\partial G$, and whose gradient belongs to the Orlicz space $L^{A}(G)$. Moreover, $L^{A_{n}}(G)$ is the optimal Orlicz range space in (1.9), in the sense that if an inequality of type (1.9) holds with $L^{A_{n}}(G)$ replaced by some other Orlicz space, then the latter must contain $L^{A_{n}}(G)$.

In particular, inequality (1.9) reproduces (1.1)-(1.3), and informs us about the fact that the spaces on the left-hand sides of (1.1)-(1.3) are the best possible in the framework of Orlicz spaces.

On the other hand, inequalities (1.1) and (1.3) can be improved if the class of admissible norms on the left-hand sides is enlarged. Indeed, if $1 \leq$ $p<n$, then there exists a constant $C$ such that

$$
\|u\|_{L^{p^{*}, p}(G)} \leq C\left|\||\nabla u|\|_{L^{p}(G)}\right.
$$

for every $u \in W_{0}^{1, p}(G)$, where $L^{p^{*}, p}(G)$ is a Lorentz space $([25,26])$. Recall that the Lorentz space $L^{p, q}(G)$, where either $1<p<\infty$ and $1 \leq q \leq \infty$, or $p=q=\infty$, is the space of all real-valued measurable functions $u$ in $G$ such that the quantity

$$
\|u\|_{L^{p, q}(G)}=\left\|s^{\frac{1}{p}-\frac{1}{q}} u^{*}(s)\right\|_{L^{q}(0,|G|)}
$$

is finite. Here, $u^{*}$ denotes the decreasing rearrangement of $u$. Inequality (1.10) is stronger than (1.1), since $L^{p^{*}, p}(G)$ is strictly contained in $L^{p^{*}}(G)$ for every $G \subset \mathbb{R}^{n}$.

The improved version of (1.3) was obtained in [8] and [21] (and can also be derived from certain capacitary estimates of [24]) and tells us that, if $|G|<\infty$, then for some positive constant $C$ and for every $u \in W_{0}^{1, n}(G)$,

$$
\|u\|_{L^{\infty, n}(\log L)^{-1}(G)} \leq C\|\| \nabla u \|_{L^{n}(G)}
$$

where $L^{\infty, n}(\log L)^{-1}(G)$ is a Lorentz-Zygmund space. 
In general, if $|G|<\infty, \alpha \in \mathbb{R}$, and either $1<p<\infty$ and $1 \leq q \leq \infty$, or $p=q=\infty$, then the Lorentz-Zygmund space $L^{p, q}(\log L)^{\alpha}(G)$ is defined as the space of all real-valued measurable functions $u$ in $G$ such that the expression

$$
\|u\|_{L^{p, q}(\log L)^{\alpha}(G)}=\left\|s^{\frac{1}{p}-\frac{1}{q}}(1+\log (|G| / s))^{\alpha} u^{*}(s)\right\|_{L^{q}(0,|G|)}
$$

is finite. Again, one can show that $L^{\infty, n}(\log L)^{-1}(G)$ is strictly contained in $\exp L^{n^{\prime}}(G)$.

The spaces $L^{p^{*}, p}(G)$ and $L^{\infty, n}(\log L)^{-1}(G)$ are known to be the best possible in inequalities (1.10) and (1.12), respectively, among all the so-called rearrangement invariant (briefly, r.i.) spaces — see [15, 18]. Loosely speaking, these are the Banach function spaces where the norm of the function depends only on its size, in the sense that it depends only on the measure of its level sets or, equivalently, on its decreasing rearrangement. Since $L^{\infty}(G)$ is contained in any other r.i. space on $G$ if $|G|<\infty$, inequality (1.2) is also the best possible as far as r.i. range spaces are concerned (although it can be strengthened on allowing norms of a different nature, such as Hölder norms, on the left-hand side). Therefore, inequalities (1.1)-(1.3) answer the question of the optimal r.i. range space in Sobolev inequalities when the domain space is $W_{0}^{1, p}(G)$.

In the present paper we solve an analogous problem in the more general context of Orlicz-Sobolev domains $W_{0}^{1, A}(G)$. Namely, given a Young function $A$, we find the smallest r.i. space into which $W_{0}^{1, A}(G)$ is continuosly embedded. The solution to this problem is provided by a space of Orlicz-Lorentz type on $G$ (or by its intersection with $L^{\infty}(G)$ ) from the family defined as follows, which includes (up to equivalent norms) the spaces $L^{p^{*}, p}(G)$ and $L^{\infty, n}(\log L)^{-1}(G)$ - see Example 1.1 below.

Definition 1.1 Let $G$ be a measurable subset of $\mathbb{R}^{n}, n \geq 1$. Let $q \in(1, \infty)$ and let $D$ be any Young function such that

$$
\int^{\infty} \frac{D(r)}{r^{1+q}} d r<\infty
$$

Then we define $L(q, D)(G)$ as the space of real-valued measurable functions $u$ on $G$ for which the quantity

$$
\|u\|_{L(q, D)(G)}=\left\|s^{-\frac{1}{q}} u^{*}(s)\right\|_{L^{D}(0,|G|)}
$$

is finite. 
Proposition 2.1, Section 2, ensures that $L(q, D)(G)$ is an r.i. space and that the expression $\|u\|_{L(q, D)(G)}$ is actually a norm on $L(q, D)(G)$. Hence, also $L(q, D)(G) \cap L^{\infty}(G)$, endowed with the norm

$$
\|u\|_{L(q, D)(G) \cap L^{\infty}(G)}=\left\|s^{-\frac{1}{q}} u^{*}(s)\right\|_{L^{D}(0,|G|)}+\|u\|_{L^{\infty}(G)},
$$

is an r.i. space on $G$. Observe that $L(q, D)(G) \cap L^{\infty}(G)$ trivially equals $L^{\infty}(G)$ whenever $|G|<\infty$.

The function $B_{A, n}$ associated with $A$ and $n$ as in the following definition plays a role in our embeddings.

Definition 1.2 Let $q \in(1, \infty)$ and let $A$ be any Young function $A$ satisfying

$$
\int_{0}\left(\frac{r}{A(r)}\right)^{\frac{1}{q-1}} d r<\infty
$$

We define the function $B_{A, q}:[0, \infty) \rightarrow[0, \infty]$ as

$$
B_{A, q}(s)=\int_{0}^{s} b(r) d r
$$

where $b$ is the left-continuous function in $[0, \infty)$ such that

$$
b^{-1}(s)=\left(\int_{a^{-1}(s)}^{\infty}\left(\int_{0}^{t}\left(\frac{1}{a(r)}\right)^{\frac{1}{q-1}} d r\right)^{-q} \frac{d t}{a(t)^{q^{\prime}}}\right)^{\frac{1}{1-q}} \text { for } s \geq 0
$$

Here, $a$ is the function appearing in (1.4), and $a^{-1}$ and $b^{-1}$ are the (generalized) left-continuous inverses of $a$ and $b$.

It turns out that $B_{A, q}$ is always a (finite-valued) Young function satisfying condition (1.14) (Proposition 2.2, Section 2), and hence $L\left(q, B_{A, q}\right)(G)$ and $L\left(q, B_{A, q}\right)(G) \cap L^{\infty}(G)$ are r.i. spaces.

Example 1.1 If $A(s)=s^{p}$, with $1 \leq p<n$, then $B_{A, n}(s)$ is equivalent to $s^{p}$, and hence

$$
L\left(n, B_{A, n}\right)(G)=L(n, A)(G)=L\left(p^{*}, p\right)(G),
$$

up to equivalent norms. If $A(s)=s^{n}$ for large $s$ and satisfies (1.6), then $B_{A, n}(s)$ is equivalent to $s^{n} \log ^{-n}(1+s)$ near infinity, and by [4, Lemma 6.12, Chapter 4]

$$
L\left(n, B_{A, n}\right)(G)=L^{\infty, n}(\log L)^{-1}(G),
$$

up to equivalent equivalent norms, provided that $|G|<\infty$. 
Our main result reads as follows.

Theorem 1.1 Let $n \geq 2$ and let $G$ be either $\mathbb{R}^{n}$, or an open subset of $\mathbb{R}^{n}$ having finite measure. Let $A$ be any Young function satisfying (1.6).

I. If

$$
\int^{\infty}\left(\frac{r}{A(r)}\right)^{\frac{1}{n-1}} d r=\infty,
$$

then there exists a constant $C_{1}$, depending only on $n$, such that

$$
\|u\|_{L\left(n, B_{A, n}\right)(G)} \leq C_{1}\||\nabla u|\|_{L^{A}(G)}
$$

for every $u \in W_{0}^{1, A}(G)$.

II. If

$$
\int^{\infty}\left(\frac{r}{A(r)}\right)^{\frac{1}{n-1}} d r<\infty
$$

then there exists a constant $C_{2}$, depending only on $n$ and $\int_{0}^{\infty} \frac{\widetilde{A}(r)}{r^{1+n^{\prime}}} d r$, such that

$$
\|u\|_{L\left(n, B_{A, n}\right)(G) \cap L^{\infty}(G)} \leq C_{2}\||\nabla u|\|_{L^{A}(G)}
$$

for every $u \in W_{0}^{1, A}(G)$. Here, $\widetilde{A}$ denotes the Young conjugate of $A$.

Moreover, $L\left(n, B_{A, n}\right)(G)$ and $L\left(n, B_{A, n}\right)(G) \cap L^{\infty}(G)$ are the optimal r.i. range spaces in inequalities (1.20) and (1.22), respectively, in the sense that if (1.20) [resp. (1.22)] holds with $L\left(n, B_{A, n}\right)(G)\left[L\left(n, B_{A, n}\right)(G) \cap L^{\infty}(G)\right]$ replaced by some other r.i. space $X(G)$, then

$$
L\left(n, B_{A, n}\right)(G) \subseteq X(G) \quad\left[L\left(n, B_{A, n}\right)(G) \cap L^{\infty}(G) \subseteq X(G)\right],
$$

with continuous inclusion.

Remark 1.1 Assumption (1.6) is indispensable for an inequality of the type

$$
\|u\|_{X\left(\mathbb{R}^{n}\right)} \leq C\||\nabla u|\|_{L^{A}\left(\mathbb{R}^{n}\right)}
$$

to hold for some r.i. space $X\left(\mathbb{R}^{n}\right)$, for some constant $\mathrm{C}$ and for every $u \in W_{0}^{1, A}\left(\mathbb{R}^{n}\right)$, as shown by Corollary 2.1 , Section 2 . When $|G|<\infty$, assumption (1.6) is irrelevant in Theorem 1.1. Actually, $A$ can be replaced, if necessary, by another Young function which is equivalent to $A$ near infinity and makes the integral in (1.6) converge. Such a replacement turns $\|\cdot\|_{L^{A}(G)}$ into an equivalent norm, up to multiplicative constants depending on $A$ and $|G|$, and hence leaves $W_{0}^{1, A}(G)$ unchanged. 
Remark 1.2 Inequality (1.20) is equivalent to the integral inequality

$$
\int_{0}^{|G|} B_{A, n}\left(C_{1}^{-1} s^{-1 / n} u^{*}(s)\right) d s \leq \int_{G} A(|\nabla u|) d x
$$

for every $u \in W_{0}^{1, A}(G)$. Indeed, (1.23) implies (1.20) by the very definition of Luxemburg norm in Orlicz spaces. Conversely, on applying (1.20) with $A(s)$ replaced by $A(s) / M$, where $M=\int_{G} A(|\nabla u|) d x$, and observing that $B_{A, n}(s)$ is transformed into $B_{A, n}(s) / M$ after this replacement, one easily obtains (1.23).

Remark 1.3 If $|G|<\infty$ and (1.21) is in force, then Theorem 1.1, Part II, reproduces the fact that $L^{\infty}(G)$ is the optimal r.i. range space for embeddings of $W_{0}^{1, A}(G)([10,29])$.

Remark 1.4 Obviously, Theorem 1.1 and inequality (1.9) tell us that either $L\left(n, B_{A, n}\right)(G)$ or $L\left(n, B_{A, n}\right)(G) \cap L^{\infty}(G)$ is (continuously) included in $L^{A_{n}}(G)$, according to whether (1.19) or (1.21) is fulfilled. However, also a direct proof of these inclusions can be accomplished, by techniques similar to those which will be used to prove the optimality of Theorem 1.1 in Section 4. This enables to recover inequality (1.9) via Theorem 1.1.

In view of Example 1.1, one is led to expect that $B_{A, n}$ is equivalent to $A$ in sub-limiting cases, but that $B_{A, n}$ grows more slowly than $A$ in borderline situations. This is actually the case. Indeed, one can show that $A$ always dominates $B_{A, n}$ (Proposition 5.1, Section 5), and that $A$ and $B_{A, n}$ are equivalent if and only if $A(s)$ is essentially below $s^{n}$. A precise statement of the latter assertion is given in Proposition 5.2, Section 5, and involves the notion of Matuzewska-Orlicz indices. Recall that if $\phi$ is a real-valued, increasing and striclty positive function in $(0, \infty)$, the upper index $I(\phi)$ of $\phi$ is defined as

$$
I(\phi)=\lim _{\lambda \rightarrow \infty} \frac{\log \left(\sup _{s>0} \frac{\phi(\lambda s)}{\phi(s)}\right)}{\log \lambda} .
$$

The upper index at infinity $I_{\infty}(\phi)$ and the upper index at zero $I_{0}(\phi)$ are defined analogously, with $\sup _{s>0}$ replaced by limsup $\sup _{s \rightarrow \infty}$ and $\lim \sup _{s \rightarrow 0+}$, respectively. The lower indices $i(\phi), i_{\infty}(\phi)$ and $i_{0}(\phi)$ are defined with $\sup _{s>0}$,

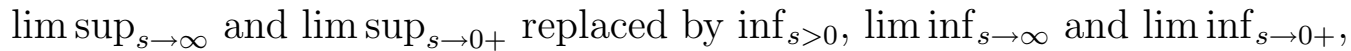
respectively. Note that $I_{0}(\phi)$ and $i_{0}(\phi)$ are well defined even if $\phi$ takes the value $\infty$. Combining Theorem 1.1 with Proposition 5.2 yields the following characterization of non-limiting optimal embeddings. 
Corollary 1.1 Let $n \geq 2$ and let $A$ be any Young satisfying (1.6).

I. Assume that (1.19) holds. Then:

(i) $L(n, A)\left(\mathbb{R}^{n}\right)$ is the optimal r.i. range space for embeddings of $W_{0}^{1, A}\left(\mathbb{R}^{n}\right)$ if and only if $A$ is finite-valued and $I(A)<n$;

(ii) Given any open set $G \subset \mathbb{R}^{n}$ having finite measure, $L(n, A)(G)$ is the optimal r.i. range space for embeddings of $W_{0}^{1, A}(G)$ into r.i. spaces if and only if $A$ is finite-valued and $I_{\infty}(A)<n$.

II. Assume that (1.21) holds. Let $\widehat{A}$ be any Young function which agrees with $A$ near 0 and satisfies $\int^{\infty} \frac{\widehat{A}(r)}{r^{1+n}} d r<\infty$. Then $L(n, \widehat{A})\left(\mathbb{R}^{n}\right) \cap L^{\infty}\left(\mathbb{R}^{n}\right)$ is the optimal r.i. range space for embeddings of $W_{0}^{1, A}\left(\mathbb{R}^{n}\right)$ if and only if $I_{0}(A)<n$.

Example 1.2 Let $G$ be an open subset of $\mathbb{R}^{n}$ having finite measure. We shall exhibit the optimal Orlicz range space and the optimal r.i. range space for embeddings of $W_{0}^{1, A}(G)$ when $A$ is a Young function which equals $s^{p} \log ^{\alpha}(1+s)$ for large $s$, where either $p=1$ and $\alpha \geq 0$, or $p>1$ and $\alpha \in \mathbb{R}$. Let us mention that special instances of this example overlap with results scattered in various papers, including $[2,3,8,10,15,17,18,19,20,21,25,26]$ Case $1 \leq p<n$. Condition (1.19) is fulfilled. The Young function $A_{n}(s)$ is equivalent to $s^{p^{*}} \log ^{\frac{n \alpha}{n-p}}(1+s)$ near infinity; thus the Orlicz space associated with this Young function agrees with the optimal Orlicz range space $L^{A_{n}}(G)$. Since $I_{\infty}(A)=p<n$, then by Corollary 1.1 the optimal r.i. space is $L(n, A)(G)$. By [4, Lemma 6.12, Chap. 4], the latter space agrees (up to equivalent norms) with the Lorentz-Zygmund space $L^{p^{*}, p}(\log L)^{\alpha / p}(G)$. Notice that this reproduces (1.10) when $\alpha=0$.

Case $p=n, \alpha<n-1$. Condition (1.19) is in force. The optimal Orlicz range space $L^{A_{n}}(G)$ equals the Orlicz space associated with the Young function

$$
e^{s^{\frac{n}{n-1-\alpha}}}-1
$$

The Young function $B_{A, n}(s)$ is equivalent to $s^{n}(\log (1+s))^{\alpha-n}$ near infinity. Thus, by $[4$, Lemma 6.12, Chap. 4], the optimal r.i.range space $L\left(n, B_{A, n}\right)(G)$ agrees with the Lorentz-Zygmund space $L^{\infty, n}(\log L)^{\alpha / n-1}(G)$. In the special case where $\alpha=0$, this recovers (1.12).

Case $p=n, \alpha=n-1$. Condition (1.19) is satisfied. The Orlicz space associated with the Young function

$$
e^{e^{s^{n^{\prime}}}}-e
$$

is the optimal Orlicz range space. 
The Young function $B_{A, n}(s)$ is equivalent to

$$
s^{n}(\log (1+s))^{-1}(\log (1+\log (1+s)))^{-n}
$$

near infinity. Hence, by a simple extension of [4, Lemma 6.12, Chap. 4], we deduce that the optimal r.i. range space $L\left(n, B_{A, n}\right)(G)$ agrees with the generalized Lorentz-Zygmund space $L^{\infty, n}(\log L)^{-1 / n}(\log \log L)^{-1}(G)$ equipped with the norm

$$
\begin{aligned}
& \|u\|_{L^{\infty, n}(\log L)^{-1 / n}(\log \log L)^{-1}(G)}= \\
& =\left\|s^{-1 / n}(1+\log (|G| / s))^{-1 / n}(1+\log (1+\log (|G| / s)))^{-1} u^{*}(s)\right\|_{L^{n}(0,|G|)} .
\end{aligned}
$$

Cases $p>n$, or $p=n$ and $\alpha>n-1$. Condition (1.21) is now fulfilled. The Young function $A_{n}(s)$ equals $\infty$ for large $s$. Thus, $L^{\infty}(G)$ is the optimal Orlicz range space, and also the optimal r.i. range space for embeddings of $W_{0}^{1, A}(G)$.

Theorem 1.1 can be extended to deal with anisotropic Orlicz-Sobolev spaces, involving Orlicz type norms depending on the full gradient of a function and not necessarily on its modulus - see e.g. [22,32]. These norms are defined by means of generalized Young functions, namely of continuous convex functions $\mathcal{A}: \mathbb{R}^{n} \rightarrow[0, \infty]$ satisfying $\mathcal{A}(0)=0, \mathcal{A}(\xi)=\mathcal{A}(-\xi)$ and such that $\left\{\xi \in \mathbb{R}^{n}: \mathcal{A}(\xi) \leq t\right\}$ is a compact set, whose interior contains 0 , for every $t>0$. The anisotropic Orlicz-Sobolev space $W_{0}^{1, \mathcal{A}}(G)$ is defined as the space of real-valued weakly differentiable functions $u$ in the open set $G$, decaying to 0 on $\partial G$, for which the quantity

$$
\|\nabla u\|_{L^{\mathcal{A}}(G)}=\inf \left\{\lambda>0: \int_{G} \mathcal{A}\left(\frac{\nabla u}{\lambda}\right) d x \leq 1\right\}
$$

is finite. Simple instances of generalized Young functions are those obeying

$$
\mathcal{A}(\xi)=\sum_{i=1}^{n} A_{i}\left(\left|\xi_{i}\right|\right) \quad \text { for } \xi \in \mathbb{R}^{n},
$$

where $A_{i}(s), i=1, \ldots, n$ are Young functions and $\xi=\left(\xi_{1}, \ldots, \xi_{n}\right)$. In particular, the choice $A_{i}(s)=s^{p_{i}}$, with $p_{i} \geq 1$, reproduces to the usual anisotropic Sobolev space (see e.g. $[5,30]$ ). A less standard example (a modification of one from [32]) is provided, for $n=2$, by the generalized Young function defined as

$$
\mathcal{A}\left(\xi_{1}, \xi_{2}\right)=\left|\xi_{1}-\xi_{2}\right|^{p_{1}}+\left|\xi_{1}\right|^{p_{2}} \log ^{\alpha}\left(k+\left|\xi_{1}\right|\right) \quad \text { for }\left(\xi_{1}, \xi_{2}\right) \in \mathbb{R}^{2},
$$

where $k$ is a sufficiently large constant, $p_{1} \geq 1$, and either $p_{2}=1, \alpha \geq 0$, or $p_{2}>1, \alpha \in \mathbb{R}$. 
Our embedding for the space $W_{0}^{1, \mathcal{A}}(G)$ makes use of the Young function $\mathcal{A}_{\star}:[0, \infty) \rightarrow[0, \infty]$ whose graph yields the profile of the graph of the symmetric rearrangement of $\mathcal{A}$. In other words, $\mathcal{A}_{\star}$ is the Young function satisfying

$$
\left|\left\{\xi \in \mathbb{R}^{n}: \mathcal{A}(\xi) \leq t\right\}\right|=\left|\left\{\xi \in \mathbb{R}^{n}: \mathcal{A}_{\star}(|\xi|) \leq t\right\}\right| \quad \text { for } t \geq 0 .
$$

In particular, it is not difficult to verify that, if $\mathcal{A}$ has the form (1.27), then $\mathcal{A}_{\star}(s)$ is equivalent to the function $\overline{\mathcal{A}}$ whose (right-continuous) inverse is given by

$$
\overline{\mathcal{A}}^{-1}(r)=\left(\prod_{i=1}^{n} A_{i}^{-1}(r)\right)^{1 / n} \text { for } r \geq 0 .
$$

The anisotropic version of Theorem 1.1 can be stated as follows.

Theorem 1.2 Let $n \geq 2$, and let $G$ be either $\mathbb{R}^{n}$, or an open subset of $\mathbb{R}^{n}$ having finite measure. Let $\mathcal{A}$ be any generalized Young function satisfying

$$
\int_{0}\left(\frac{r}{\mathcal{A}_{\star}(r)}\right)^{\frac{1}{n-1}} d r<\infty .
$$

Let $B_{\mathcal{A}_{\star}, n}$ be given as in Definition 1.2, with $A$ replaced by $\mathcal{A}_{\star}$.

I. If

$$
\int^{\infty}\left(\frac{r}{\mathcal{A}_{\star}(r)}\right)^{\frac{1}{n-1}} d r=\infty,
$$

then there exists a constant $C_{1}$, depending only on $n$, such that

$$
\|u\|_{\left.L\left(n, B_{\mathcal{A}_{\star}, n}\right)\right)(G)} \leq C_{1}\|\nabla u\|_{L^{\mathcal{A}}(G)}
$$

for every $u \in W_{0}^{1, \mathcal{A}}(G)$.

II. If

$$
\int^{\infty}\left(\frac{r}{\mathcal{A}_{\star}(r)}\right)^{\frac{1}{n-1}} d r<\infty
$$

then there exists a constant $C_{2}$, depending only on $n$ and $\int_{0}^{\infty} \frac{\widetilde{\mathcal{A}_{\star}}(r)}{r^{1+n^{\prime}}} d r$, such that

$$
\|u\|_{L\left(n, B_{\mathcal{A}_{\star}, n}\right)(G) \cap L^{\infty}(G)} \leq C_{2}\|\nabla u\|_{L^{\mathcal{A}_{\star}(G)}}
$$

for every $u \in W_{0}^{1, \mathcal{A}}(G)$.

Moreover, $L\left(n, B_{\mathcal{A}_{\star}, n}\right)(G)$ and $L\left(n, B_{\mathcal{A}_{\star}, n}\right)(G) \cap L^{\infty}(G)$ are the optimal r.i. range spaces in inequalities (1.32) and (1.34), respectively, in the sense that if (1.32) [resp. (1.34)] holds, for every generalized Young function $\mathcal{A}$ having prescribed $\mathcal{A}_{\star}$, with $L\left(n, B_{\mathcal{A}_{\star}, n}\right)(G)\left[L\left(n, B_{\mathcal{A}_{\star}, n}\right)(G) \cap L^{\infty}(G)\right]$ replaced by some other r.i. space $X(G)$, then $\left.L\left(n, B_{\mathcal{A}_{\star}, n}\right)\right)(G) \subseteq X(G)\left[L\left(n, B_{\mathcal{A}_{\star}, n}\right)(G) \cap\right.$ $\left.L^{\infty}(G) \subseteq X(G)\right]$, with continuous inclusion. 
Remarks analogous to those following Theorem 1.1 hold for Theorem 1.2. Moreover, an inspection of the proof will reveal that $\mathcal{A}_{\star}$ may be replaced in the statement of Theorem 1.2 by any equivalent Young function if $G=\mathbb{R}^{n}$, and by any equivalent Young function near infinity if $|G|<\infty$. In analogy with Corollary 1.1, a description of non-limiting situations in terms of the indices of $\mathcal{A}_{\star}$ can be provided. Furthermore, inclusions like those described in Remark 1.4, involving $\mathcal{A}_{\star}$ instead of $A$, may be proved; hence, one can recover the optimal embedding, established in [14], for $W_{0}^{1, \mathcal{A}}(G)$ into the Orlicz space associated with the Young function $\left(\mathcal{A}_{\star}\right)_{n}$ defined as in (1.7)-(1.8), with $A$ replaced by $\mathcal{A}_{\star}$.

Example 1.3 Let $G$ be an open subset of $\mathbb{R}^{n}$. Assume that $\mathcal{A}$ has the form (1.27) with $A_{i}(s)=s^{p_{i}}, p_{i} \geq 1$. The function $\mathcal{A}_{\star}(s)$ is then equivalent to $\overline{\mathcal{A}}$, defined by (1.29), and $\overline{\mathcal{A}}(s)=s^{\bar{p}}$, where $\frac{1}{\bar{p}}=\frac{1}{n} \sum_{i=1}^{n} \frac{1}{p_{i}}$. Thus, on making use of Theorem 1.2 and of the subsequent remarks, and proceeding as in Example 1.2, the following conclusions can be derived about optimal embeddings of $W_{0}^{1, \mathcal{A}}(G)$ as $\mathcal{A}$ ranges among all generalized Young functions with $\mathcal{A}_{\star}(s)$ equivalent to $s^{\bar{p}}$.

Case $\sum_{i=1}^{n} \frac{1}{p_{i}}>1$. $L^{\bar{p}^{*}}(G)$ is the optimal Orlicz range space (see $\left.[5,30]\right)$, and $L\left(\bar{p}^{*}, \bar{p}\right)(G)$ is the optimal r.i. range space $([5,23,28])$.

Case $\sum_{i=1}^{n} \frac{1}{p_{i}}=1$. If $|G|<\infty$, then $\exp L^{n^{\prime}}(G)$ is the optimal Orlicz range space $([22,28])$, and $L^{\infty, n}(\log L)^{-1}(G)$ is the optimal r.i. range space.

Case $\sum_{i=1}^{n} \frac{1}{p_{i}}<1$. If $|G|<\infty$, then $L^{\infty}(G)$ is the optimal Orlicz and r.i. range space $([5,28])$.

Example 1.4 Let $G$ be an open subset of $\mathbb{R}^{2}$ having finite measure. Consider the generalized Young function $\mathcal{A}$ given by (1.28). It is not difficult to verify that $\mathcal{A}_{\star}(s)$ is equivalent to

$$
s^{\frac{2 p_{1} p_{2}}{p_{1}+p_{2}}} \log \frac{\alpha p_{1}}{p_{1}+p_{2}}(1+s)
$$

near infinity (see e.g. [14]). Thus, Theorem 1.2 and the subsequent remarks, together with considerations similar to those of Example 1.2, yield the following information about optimal embeddings of $W_{0}^{1, \mathcal{A}}(G)$, when $\mathcal{A}$ is any generalized Young function with $\mathcal{A}_{\star}(s)$ equivalent to

$$
s^{\frac{2 p_{1} p_{2}}{p_{1}+p_{2}}} \log ^{\frac{\alpha p_{1}}{p_{1}+p_{2}}}(1+s)
$$

near infinity. 
Case $p_{1} p_{2}<p_{1}+p_{2}$. The optimal Orlicz range space is generated by the Young function

$$
s^{\frac{2 p_{1} p_{2}}{p_{1}+p_{2}-p_{1} p_{2}}} \log \frac{\alpha p_{1}}{\overline{p_{1}+p_{2}-p_{1} p_{2}}}(1+s) .
$$

The optimal r.i. range space agrees with the Lorentz-Zygmund space

$$
L^{\frac{2 p_{1} p_{2}}{p_{1}+p_{2}-p_{1} p_{2}}}, \frac{2 p_{1} p_{2}}{p_{1}+p_{2}}(\log L)^{\frac{\alpha}{2 p_{2}}}(G) .
$$

Case $p_{1} p_{2}=p_{1}+p_{2}, \alpha p_{1}<p_{1}+p_{2}$. The optimal Orlicz range space is generated by the Young function

$$
e^{s^{\frac{2\left(p_{1}+p_{2}\right)}{p_{1}+p_{2}-\alpha p_{1}}}}-1
$$

The optimal r.i. range space is $L^{\infty, 2}(\log L)^{\frac{\alpha p_{1}}{2\left(p_{1}+p_{2}\right)}-1}(G)$.

Case $p_{1} p_{2}=p_{1}+p_{2}, \alpha p_{1}=p_{1}+p_{2}$. The space associated with the Young function $e^{e^{s^{2}}}-e$ is the optimal Orlicz range space. The optimal r.i. range space agrees with the generalized Lorentz-Zygmund space

$$
L^{\infty, 2}(\log L)^{-1 / 2}(\log \log L)^{-1}(G)
$$

Cases $p_{1} p_{2}>p_{1}+p_{2}$, or $p_{1} p_{2}=p_{1}+p_{2}$ and $\alpha p_{1}>p_{1}+p_{2}$. The space $L^{\infty}(G)$ is the optimal Orlicz and r.i. range space.

\section{Preliminaries}

\subsection{Rearrangements and rearrangement invariant spaces}

Let $G$ be a measurable subset of $\mathbb{R}^{n}$ and let $u$ be a real-valued measurable function in $G$. The decreasing rearrangement $u^{*}$ of $u$ is the function from $[0, \infty)$ into $[0, \infty]$ defined as

$$
u^{*}(s)=\sup \{t \geq 0:|\{x \in G:|u(x)|>t\}|>s\} \quad \text { for } s \geq 0 .
$$

It is easily verified that $u^{*}$ is the unique non-increasing, right-continuous function in $[0, \infty)$ which is equidistributed with $u$. Notice that $[0,|G|] \supseteq$ $\operatorname{supp} u^{*}$, the support of $u^{*}$.

Given $u \in L_{l o c}^{1}(G)$, the elementary maximal function $u^{* *}$ associated with $u^{*}$ is defined as

$$
u^{* *}(s)=\frac{1}{s} \int_{0}^{s} u^{*}(r) d r \quad \text { for } s>0 .
$$


Since $u^{*}$ is non-increasing, $u^{* *}$ is also non-increasing and $u^{*} \leq u^{* *}$. Recall that if $u, v \in L_{l o c}^{1}(G)$, then

$$
(u+v)^{* *}(s) \leq u^{* *}(s)+v^{* *}(s) \quad \text { for } s>0 .
$$

Moreover,

(2.4) if $u^{* *}(s) \leq v^{* *}(s)$ for $s>0$, then $\int_{0}^{\infty} u^{*}(s) g(s) d s \leq \int_{0}^{\infty} v^{*}(s) g(s) d s$ for every non-increasing function $g:[0, \infty) \rightarrow[0, \infty)$.

A basic property of rearrangements is the Hardy-Littlewood inequality, which tells us that

$$
\int_{G}|u v| d x \leq \int_{0}^{|G|} u^{*}(r) v^{*}(r) d r
$$

for all measurable functions $u$ and $v$ in $G$.

A set $X(G)$ of real-valued measurable functions in $G$ is called a rearrangement invariant Banach function space if it is a linear space equipped with a norm $\|\cdot\|_{X(G)}$ satisfying the following properties:

(P1) if $0 \leq v \leq u$ a.e. in $G$ and $u \in X(G)$, then $v \in X(G)$ and $\|v\|_{X(G)} \leq$ $\|u\|_{X(G)}$

(P2) if $u_{n}$ is a sequence such that $0 \leq u_{n} \nearrow u$ a.e. in $G$ and $u \in X(G)$, then $\left\|u_{n}\right\|_{X(G)} \nearrow\|u\|_{X(G)}$;

(P3) $\left\|\chi_{E}\right\|_{X(G)}<\infty$ for every $E \subseteq G$ such that $|E|<\infty$; here, $\chi_{E}$ denotes the characteristic function of the set $E$;

(P4) for every $E \subseteq G$ with $|E|<\infty$, there exists a constant $C$ such that $\int_{E} u d x \leq C\|u\|_{X(G)}$ for all $u \in X(G)$;

(P5) if $u \in X(G)$ and $v^{*}=u^{*}$, then $v \in X(G)$ and $\|v\|_{X(G)}=\|u\|_{X(G)}$.

The associate space $X^{\prime}(G)$ of $X(G)$ is defined as

$$
\begin{array}{r}
X^{\prime}(G)=\{v: v \text { is a real-valued measurable function in } G \\
\text { and } \left.\int_{G}|u v| d x<\infty \text { for all } u \in X(G)\right\},
\end{array}
$$

and is an r.i. space endowed with the norm

$$
\|v\|_{X^{\prime}(G)}=\sup _{u \neq 0} \frac{\int|u v| d x}{\|u\|_{X(G)}} .
$$


For every r.i. space $X(G)$,

$$
\left(X^{\prime}\right)^{\prime}(G)=X(G)
$$

with equal norms. The Hölder type inequality

$$
\int_{G}|u v| d x \leq\|u\|_{X(G)}\|v\|_{X^{\prime}(G)}
$$

holds for every $u \in X(G)$ and $v \in X^{\prime}(G)$, and is an obvious consequence of definition (2.6).

The representation space $\bar{X}(0,|G|)$ of $X(G)$ is the unique r.i. space on $[0,|G|]$ satisfying

$$
\|u\|_{X(G)}=\left\|u^{*}\right\|_{\bar{X}(0,|G|)}
$$

for every $u \in X(G)$. It is equipped with the norm

$$
\|f\|_{\bar{X}(0,|G|)}=\sup _{\|v\|_{X^{\prime}(G)} \leq 1} \int_{0}^{|G|} f^{*}(r) v^{*}(r) d r .
$$

A property of r.i. spaces states that if $X_{1}(G)$ and $X_{2}(G)$ are r.i. spaces, then

$$
X_{1}(G) \subseteq X_{2}(G) \text { if and only if }\|u\|_{X_{2}(G)} \leq C\|u\|_{X_{1}(G)}
$$

for some positive constant $C$ and for every $u \in X_{1}(G)$.

For a detailed treatment of the theory of r.i. spaces, we refer to [4].

Customary examples of r.i. spaces are provided by Lebesgue, Orlicz, Lorentz and (generalized) Lorentz-Zygmund spaces, whose definition has already been reproduced in Section 1. Below, some more facts about these spaces are recalled.

The theory of Orlicz spaces relies on properties of Young functions. The following properties will come into play in our discussion. Given any Young function $A$, we have:

$$
A(s) \leq s a(s) \leq 2 A(2 s) \quad \text { for } s \geq 0,
$$

where $a$ is the function appearing in (1.4);

$$
\begin{aligned}
k A(s) & \leq A(k s) \quad \text { for } k \geq 1 \text { and } s \geq 0 \\
A\left(s_{1}\right)+A\left(s_{2}\right) & \leq A\left(s_{1}+s_{2}\right) \quad \text { for } s_{1}, s_{2} \geq 0 .
\end{aligned}
$$


The Young conjugate $\widetilde{A}$ of $A$ is the Young function defined by $\widetilde{A}(s)=$ $\sup \{r s-A(r): r \geq 0\}$ for $s \geq 0$, and satisfies

$$
\widetilde{A}(s)=\int_{0}^{s} a^{-1}(r) d r \quad \text { for } s \geq 0
$$

where $a^{-1}$ is the (generalized) left-continuous inverse of $a$. Notice that $\widetilde{\widetilde{A}}=A$. Moreover,

$$
r \leq A^{-1}(r) \widetilde{A}^{-1}(r) \leq 2 r \quad \text { for } r \geq 0,
$$

where $A^{-1}$ and $\widetilde{A}^{-1}$ are (generalized) right-continuous inverses.

The Orlicz space $L^{A}(G)$ is an r.i. space equipped with the Luxemburg norm defined in (1.5). Its representation space is $L^{A}(0,|G|)$, and its associate space is, up to equivalent norms, $L^{\widetilde{A}}(G)$. Actually, the inequalities

$$
\|u\|_{L^{\tilde{A}(G)}} \leq\|u\|_{\left(L^{A}\right)^{\prime}(G)} \leq 2\|u\|_{L^{\tilde{A}}(G)}
$$

hold for every $u \in L^{\widetilde{A}}(G)$.

A function $\phi:[0, \infty) \rightarrow[0, \infty]$ is said to dominate another function $\psi:[0, \infty) \rightarrow[0, \infty]$ globally if a positive constant $c$ exists such that $\psi(s) \leq$ $\phi(c s)$ for $s \geq 0$. The function $\phi$ is said to dominate $\psi$ near infinity [resp. near 0] if positive constants $c$ and $s_{\infty}\left[s_{0}\right]$ exist such that (1.22) holds for $s \geq s_{\infty}\left[0 \leq s \leq s_{0}\right]$. The functions $\phi$ and $\psi$ are called globally equivalent if they dominate each other globally; a corresponding definition is given for functions equivalent near infinity or near 0 . Given two Young functions $A$ and $B$, the continuous inclusion $L^{A}(G) \subseteq L^{B}(G)$ holds if and only if either $|G|=\infty$ and $A$ dominates $B$ globally, or $|G|<\infty$ and $A$ dominates $B$ near infinity.

The quantities $\|\cdot\|_{L^{p, q}(G)}$ and $\|\cdot\|_{L^{p, q}(\log L)^{\alpha}(G)}$, given by (1.11) and (1.13), are in general only quasi-norms on the Lorentz and the Lorentz-Zygmund spaces, respectively, since they may fail to satisfy the triangle inequality. They can be turned into equivalent norms on replacing $u^{*}$ by $u^{* *}$ in definitions (1.11) and (1.13). In the special cases when the weights $s^{1 / p-1 / q}$ or $s^{1 / p-1 / q}(\log (1+|G| / s))^{\alpha}$ are non-increasing (and hence in all the cases involved in the embeddings discussed in Section 1), also $\|\cdot\|_{L^{p, q}(G)}$ and $\|\cdot\|_{L^{p, q}(\log L)^{\alpha}(G)}$ are norms. The representation spaces of $L^{p, q}(G)$ and $L^{p, q}(\log L)^{\alpha}(G)$ are $L^{p, q}(0,|G|)$ and $L^{p, q}(\log L)^{\alpha}(0,|G|)$, respectively. Analogous considerations hold for generalized Lorentz-Zygmund spaces. Notice the alternative formula

$$
\|u\|_{L^{p, q}(G)}=\left\{\begin{array}{cl}
\left(q \int_{0}^{\infty} t^{q-1}|\{x \in G:|u(x)|>t\}|^{q / p} d t\right)^{1 / q} & \text { if } q<\infty \\
\sup _{t>0} t|\{x \in G:|u(x)|>t\}|^{1 / p} & \text { if } q=\infty
\end{array}\right.
$$




\subsection{Orlicz-Lorentz spaces}

This subsection is devoted to the study of the spaces $L(q, D)(G)$ of OrliczLorentz type introduced in Definition 1.1, and of the function $B_{A, q}$ associated with $A$ and $q$ as in Definition 1.2. Our first result states that $L(q, D)(G)$ is actually an r.i. space.

Proposition 2.1 Let $G$ be any measurable subset of $\mathbb{R}^{n}, n \geq 1$. Let $q \in$ $(1, \infty)$ and let $D$ be a Young function satisfying (1.14). Then:

I. The space $L(q, D)(G)$, equipped with the norm (1.15), is an r.i. space.

II. The space $L(q, D)(G) \cap L^{\infty}(G)$, equipped with the norm (1.16), is an r.i. space. Moreover, if we define the function $\varphi_{q}:[0, \infty) \rightarrow[0, \infty)$ as

$$
\varphi_{q}(s)=\min \left\{1, s^{-1 / q}\right\} \quad \text { for } s>0,
$$

and the function $E_{D}:[0, \infty) \rightarrow[0, \infty]$ as

$$
E_{D}(t)= \begin{cases}D(t) & \text { if } 0 \leq t \leq 1 \\ \infty & \text { if } t>1\end{cases}
$$

then the space $L(q ; D, \infty)(G)$ of those real-valued measurable functions $u$ in $G$ for which the quantity

$$
\|u\|_{L(q ; D, \infty)(G)}=\inf \left\{\lambda>0: \int_{0}^{\infty} E_{D}\left(\frac{\varphi_{q}(s) u^{*}(s)}{\lambda}\right) d s \leq 1\right\}
$$

is finite is an r.i. space equipped with the norm $\|\cdot\|_{L(q ; D, \infty)(G)}$, and

$$
L(q, D)(G) \cap L^{\infty}(G)=L(q ; D, \infty)(G),
$$

up to equivalent norms.

Proof. Part I. We have first to check that $\|\cdot\|_{L(q, D)(G)}$ is a norm. The only nontrivial property of norms to be verified is the triangle inequality. We have

$$
\begin{aligned}
& \| u+v\left\|_{L(q, D)(G)}=\right\| s^{-1 / q}(u+v)^{*}(s) \|_{L^{D}(0,|G|)} \\
&=\sup _{w \in\left(L^{D}\right)^{\prime}(G)} \frac{\int_{0}^{\infty} s^{-1 / q}(u+v)^{*}(s) w^{*}(s) d s}{\|w\|_{\left(L^{D}\right)^{\prime}(G)}} \\
& \leq \sup _{w \in\left(L^{D}\right)^{\prime}(G)} \frac{\int_{0}^{\infty} s^{-1 / q} u^{*}(s) w^{*}(s) d s+\int_{0}^{\infty} s^{-1 / q} v^{*}(s) w^{*}(s) d s}{\|w\|_{\left(L^{D}\right)^{\prime}(G)}} \\
& \leq \sup _{w \in\left(L^{D}\right)^{\prime}(G)} \frac{\int_{0}^{\infty} s^{-1 / q} u^{*}(s) w^{*}(s) d s}{\|w\|_{\left(L^{D}\right)^{\prime}(G)}}+\sup _{w \in\left(L^{D}\right)^{\prime}(G)} \frac{\int_{0}^{\infty} s^{-1 / q} v^{*}(s) w^{*}(s) d s}{\|w\|_{\left(L^{D}\right)^{\prime}(G)}} \\
&=\left\|s^{-1 / q} u^{*}(s)\right\|_{L^{D}(0,|G|)}+\left\|s^{-1 / q} v^{*}(s)\right\|_{L^{D}(0,|G|)}=\|u\|_{L(q, D)(G)}+\|v\|_{L(q, D)(G)},
\end{aligned}
$$

where the second and third equalities are due to (2.7) and the first inequality to $(2.3)-(2.4)$. 
Consider now properties (P1)-(P5) of the definition of r.i. spaces. Property (P1) holds thanks to the corresponding property for $L^{D}(0,|G|)$, since decreasing rearrangement preserves pointwise a.e. inequalities between nonnegative functions.

Property (P2) is a consequence of the corresponding property of $L^{D}(0,|G|)$ and of the fact that if $0 \leq u_{n} \nearrow u$ a.e., then $0 \leq u_{n}^{*} \nearrow u^{*}$ everywhere (see [4, Prop. 1.7, Chap. 2]).

Assumption (1.14) is equivalent to requiring that

$$
\left\|s^{-1 / q} \chi_{(0,|E|)}(s)\right\|_{L^{D}(0,|G|)}<\infty
$$

whenever $|E|<\infty$, whence (P3) follows.

Property (P4) holds since, by (2.5) and (2.16),

$$
\begin{aligned}
\int_{E}|u| d x & \leq \int_{0}^{|E|} u^{*}(s) d s \leq 2\left\|s^{-1 / q} u^{*}(s)\right\|_{L^{D}(0,|G|)}\left\|s^{1 / q} \chi_{(0,|E|)}(s)\right\|_{L^{\tilde{D}}(0,|G|)} \\
& \leq \frac{2|E|^{1 / q}}{\widetilde{D}^{-1}(1 /|E|)}\|u\|_{L(q, D)(G)}
\end{aligned}
$$

Property (P5) is obvious.

Part II. As an intersection of r.i. spaces, $L(q, D)(G) \cap L^{\infty}(G)$ is also an r.i. space endowed with the norm (1.16). The proof of the fact that $L(q ; D, \infty)(G)$ is an r.i. space parallels that of Part I. When $|G|<\infty$, equation $(2.21)$ is trivial, since both $L(q, D)(G) \cap L^{\infty}(G)$ and $L(q ; D, \infty)(G)$ equal $L^{\infty}(G)$. The proof of equation $(2.21)$ when $|G|=\infty$ is omitted for brevity: it just makes use of arguments based on the very definitions of the norms (1.16) and (2.20).

The fact that the space $L_{q, B_{A, q}}(G)$ is always an r.i. space is a consequence of Proposition 2.1 and of the following proposition.

Proposition 2.2 Let $q \in(1, \infty)$ and let $A$ be any Young function satisfying (1.17). Then the function $B_{A, q}$ is a finite-valued Young function and

$$
\int^{\infty} \frac{B_{A, q}(r)}{r^{1+q}} d r<\infty
$$

Proof. In order to prove that $B_{A, q}$ is a Young function, we need only to show that the function $b^{-1}$, given by (1.18), is neither identically equal to 0 , nor to $\infty$. Since $A$ is a Young function, then $a$ is neither identically 0 nor identically $\infty$; hence, the same property is enjoyed by $a^{-1}$. Thus, it suffices to verify that

$$
\int_{0}\left(\frac{1}{a(r)}\right)^{\frac{1}{q-1}} d r<\infty
$$


and

$$
\int^{\infty}\left(\int_{0}^{t}\left(\frac{1}{a(r)}\right)^{\frac{1}{q-1}} d r\right)^{-q} \frac{d t}{a(t)^{q^{\prime}}}<\infty .
$$

Condition (2.23) is equivalent to (1.17), by Lemma 2.3 below. Concerning (2.24), we have

$$
\begin{aligned}
\int_{1}^{\infty}\left(\int_{0}^{t}\left(\frac{1}{a(r)}\right)^{\frac{1}{q-1}} d r\right)^{-q} \frac{d t}{a(t)^{q^{\prime}}} & \leq \frac{1}{a(1)} \int_{1}^{\infty}\left(\int_{0}^{t}\left(\frac{1}{a(r)}\right)^{\frac{1}{q-1}} d r\right)^{-q} \frac{1}{a(t)^{q^{\prime}-1}} d t \\
& \leq \frac{1}{(1-q) a(1)}\left(\int_{0}^{1}\left(\frac{1}{a(r)}\right)^{\frac{1}{q-1}} d r\right)^{1-q} .
\end{aligned}
$$

Thus, (2.24) holds. Condition (2.22) is a consequence of Lemmas 2.3-2.4 below.

Lemma 2.3 Let $q \in(1, \infty)$ and let $A$ be a Young function.

I. The following conditions are equivalent:
(i) $\int^{\infty} \frac{A(r)}{r^{1+q}} d r<\infty$;
(ii) $\int^{\infty} \frac{a(r)}{r^{q}} d r<\infty$;
(iii) $\int^{\infty}\left(\frac{1}{a^{-1}(r)}\right)^{q-1} d r<\infty$;
(iv) $\int^{\infty}\left(\frac{r}{\widetilde{A}(r)}\right)^{q-1} d r<\infty$.

II. The following conditions are equivalent:
(i) $\int_{0} \frac{A(r)}{r^{1+q}} d r<\infty$
(ii) $\int_{0} \frac{a(r)}{r^{q}} d r<\infty$;
(iii) $\int_{0}\left(\frac{1}{a^{-1}(r)}\right)^{q-1} d r<\infty$;
(iv) $\int_{0}\left(\frac{r}{\widetilde{A}(r)}\right)^{q-1} d r<\infty$.

Proof. Part I. The equivalence of (i) and (ii) is due to (2.11); since $\widetilde{A}$ and $a^{-1}$ are related by (2.14), the equivalence of (iii) and (iv) follows analogously. The equivalence of (ii) and (iii) is a consequence of the fact that, if any of the two conditions is satisfied, then $a$ must be finite-valued and, by Fubini's theorem,

$$
\begin{aligned}
\int_{1}^{\infty} \frac{a(r)}{r^{q}} d r & =\int_{0}^{a(1)} \int_{1}^{\infty} r^{-q} d r d \tau+\int_{a(1)}^{\infty} \int_{a^{-1}(\tau)}^{\infty} r^{-q} d r d \tau \\
& =\frac{a(1)}{q-1}+\frac{1}{q-1} \int_{a(1)}^{\infty}\left(\frac{1}{a^{-1}(\tau)}\right)^{q-1} d \tau
\end{aligned}
$$

The proof of Part II is analogous. 
Lemma 2.4 Let $q \in(1, \infty)$ and let $A$ be any Young function satisfying (1.17). Set

$$
\sigma(s)=a^{-1}(b(s)) \quad \text { for } s>0,
$$

where $b$ is as in Definition 1.2. Then

$$
\left(\int_{s}^{\infty} \frac{b(r)}{r^{q}} d r\right)^{1 / q}\left(\int_{0}^{\sigma(s)}\left(\frac{1}{a(r)}\right)^{\frac{1}{q-1}} d r\right)^{1 / q^{\prime}} \leq \frac{1}{(q-1)^{2 / q}} \text { for } s>0
$$

and

$$
\frac{b(s)}{s^{q-1}}\left(\int_{0}^{\sigma(s)}\left(\frac{1}{a(r)}\right)^{\frac{1}{q-1}} d r\right)^{q-1} \leq \frac{1}{q-1} \text { for } s>0 .
$$

Proof. We have

$$
\begin{aligned}
(q-1) & \int_{s}^{\infty} \frac{b(t)}{t^{q}} d t=(q-1) \int_{s}^{\infty} t^{-q} \int_{0}^{b(t)} d \tau d t \\
= & (q-1)\left(\int_{0}^{b(s)} \int_{s}^{\infty} t^{-q} d t d \tau+\int_{b(s)}^{\infty} \int_{b^{-1}(\tau)}^{\infty} t^{-q} d t d \tau\right) \\
= & \frac{b(s)}{s^{q-1}}+\int_{b(s)}^{\infty}\left(\frac{1}{b^{-1}(\tau)}\right)^{1-q} d \tau \\
= & \frac{b(s)}{s^{q-1}}+\int_{b(s)}^{\infty}\left(\int_{a^{-1}(\tau)}^{\infty}\left(\int_{0}^{t}\left(\frac{1}{a(r)}\right)^{\frac{1}{q-1}} d r\right)^{-q} \frac{d t}{a(t)^{q^{\prime}}}\right) d \tau \\
= & \frac{b(s)}{s^{q-1}}+\int_{a^{-1}(b(s))}^{\infty}\left(\int_{0}^{t}\left(\frac{1}{a(r)}\right)^{\frac{1}{q-1}} d r\right)^{-q} \frac{a(t)-b(s)}{a(t)^{q^{\prime}}} d t \\
= & b(s)\left[\frac{1}{s^{q-1}}-\int_{a^{-1}(b(s))}^{\infty}\left(\int_{0}^{t}\left(\frac{1}{a(r)}\right)^{\frac{1}{q-1}} d r\right)^{-q} \frac{1}{a(t)^{q^{\prime}}} d t\right] \\
& +\int_{a^{-1}(b(s))}^{\infty}\left(\int_{0}^{t}\left(\frac{1}{a(r)}\right)^{\frac{1}{q-1}} d r\right)^{-q} \frac{1}{a(t)^{\frac{1}{q-1}}} d t \\
= & b(s)\left[\frac{1}{s^{q-1}}-\frac{1}{\left(b^{-1}(b(s))\right)^{q-1}}\right]+\frac{1}{q-1}\left[\left(\int_{0}^{\sigma(s)}\left(\frac{1}{a(r)}\right)^{\frac{1}{q-1}} d r\right)^{1-q}\right. \\
& \left.-\left(\int_{0}^{\infty}\left(\frac{1}{a(r)}\right)^{\frac{1}{q-1}} d r\right)^{1-q}\right]
\end{aligned}
$$

for $s>0$. Hence,

$$
(q-1) \int_{s}^{\infty} \frac{b(t)}{t^{q}} d t \leq \frac{1}{q-1}\left(\int_{0}^{\sigma(s)}\left(\frac{1}{a(r)}\right)^{\frac{1}{q-1}} d r\right)^{1-q} \quad \text { for } s>0,
$$


and (2.26) follows. Since

$$
\int_{s}^{\infty} \frac{b(t)}{t^{q}} d t \geq b(s) \frac{s^{1-q}}{q-1} \quad \text { for } s>0
$$

inequality (2.28) also implies (2.27).

\subsection{Sobolev type spaces and inequalities}

Let $G$ be an open subset of $\mathbb{R}^{n}$ and let $A$ be a Young function. We denote by $W_{0}^{1, A}(G)$ the Orlicz-Sobolev space of those weakly differentiable functions in $G$, with $\||\nabla u|\|_{L^{A}(G)}<\infty$, which decay to 0 on $\partial G$, in the sense that the continuation of $u$ by 0 outside $G$ is a weakly differentiable function in $\mathbb{R}^{n}$ and

$$
|\{x \in G:|u(x)|>t\}|<\infty \quad \text { for every } t>0 \text {. }
$$

Obviously, condition (2.29) is automatically satisfied if $|G|<\infty$. Given a generalized Young function $\mathcal{A}$, the anisotropic Orlicz-Sobolev space $W_{0}^{1, \mathcal{A}}(G)$ is defined analogously, by requiring that $\|\nabla u\|_{L^{\mathcal{A}}(G)}<\infty$.

A basic tool in our applications to Sobolev type inequalities is the PólyaSzegö principle on the decrease of gradient norms under rearrangement. In its version for Orlicz norms, such a principle tells us that if $A$ is any Young function and $u \in W_{0}^{1, A}(G)$, then $u^{*}$ is locally absolutely continuous in $(0, \infty)$, and

$$
\left\|n \omega_{n}{ }^{1 / n} r^{1 / n^{\prime}}\left(-\frac{d u^{*}}{d r}\right)\right\|_{L^{A}(0,|G|)} \leq\||\nabla u|\|_{L^{A}(G)}
$$

(see e.g. [9]). In particular, equality holds in (2.30) whenever $u$ is spherically symmetric. A version of this principle for anisotropic Orlicz norms is also available $([22])$. It implies that if $\mathcal{A}$ is any generalized Young function and $u \in W_{0}^{1, \mathcal{A}}(G)$, then $u^{*}$ is locally absolutely continuous in $(0, \infty)$ and a constant $K(n)$, depending only on $n$, exists such that

$$
\left\|K(n) r^{1 / n^{\prime}}\left(-\frac{d u^{*}}{d r}\right)\right\|_{L^{\mathcal{A}_{\star}(0,|G|)}} \leq\|\nabla u\|_{L^{\mathcal{A}}(G)},
$$

where $\mathcal{A}_{\star}$ is the Young function defined in Section 1.

Inequalities (2.30) - (2.31) enable to reduce the problem of Orlicz-Sobolev embeddings into r.i. spaces to one-dimensional inequalities for a Hardy type operator. This is the content of the following proposition, our starting point in the proofs of Theorems 1.1 and 1.2. 
Proposition 2.5 Let $n \geq 2$ and let $G$ be either $\mathbb{R}^{n}$, or an open subset of $\mathbb{R}^{n}$ having finite measure. Let $X(G)$ be an r.i. space on $G$.

I. Let $A$ be a Young function. Then the following assertions are equivalent:

(i) A constant $K_{1}$ exists such that

$$
\|u\|_{X(G)} \leq K_{1}\||\nabla u|\|_{L^{A}(G)} \quad \text { for every } u \in W_{0}^{1, A}(G) .
$$

(ii) A constant $K_{2}$ exists such that

$$
\left\|\int_{s}^{|G|} r^{-1 / n^{\prime}} f(r) d r\right\|_{\bar{X}(0,|G|)} \leq K_{2}\|f\|_{L^{A}(0,|G|)}
$$

for every $f \in L^{A}(0,|G|)$.

II. The following assertions are equivalent:

(i) For any generalized Young function $\mathcal{A}$, with prescribed $\mathcal{A}_{\star}$, a constant $K_{1}$ exists such that

$$
\|u\|_{X(G)} \leq K_{1}\|\nabla u\|_{L^{\mathcal{A}}(G)} \quad \text { for every } u \in W_{0}^{1, \mathcal{A}}(G) .
$$

(ii) A constant $K_{2}$ exists such that

$$
\left\|\int_{s}^{|G|} r^{-1 / n^{\prime}} f(r) d r\right\|_{\bar{X}(0,|G|)} \leq K_{2}\|f\|_{L^{\mathcal{A}_{\star}(0,|G|)}}
$$

for every $f \in L^{\mathcal{A}_{\star}}(0,|G|)$.

As for the proof of Proposition 2.5, the fact that (ii) implies (i) in Part I is an easy consequence of (2.30), whereas the converse implication can be established on choosing spherically symmetric test functions in (2.32). The proof of Part II is analogous: one has to make use of (2.31) instead of (2.30) for one implication, and to choose $\mathcal{A}(\xi)=\mathcal{A}_{\star}(|\xi|)$ and spherically symmetric test functions in (2.33) for the reverse implication. The details are omitted for brevity. As a first consequence of Proposition 2.5, the necessity of condition (1.6) or (1.30) for inequalities in $\mathbb{R}^{n}$ can be derived.

Corollary 2.1 Let $n \geq 2$ and let $X\left(\mathbb{R}^{n}\right)$ be any r.i. space on $\mathbb{R}^{n}$.

I. Assume that a constant $C$ exists such that

$$
\|u\|_{X\left(\mathbb{R}^{n}\right)} \leq C\||\nabla u|\|_{L^{A}\left(\mathbb{R}^{n}\right)}
$$

for some Young function $A$ and for every $u \in W_{0}^{1, A}\left(\mathbb{R}^{n}\right)$. Then $A$ satisfies (1.6).

II. Assume that a constant $C$ exists such that

$$
\|u\|_{X\left(\mathbb{R}^{n}\right)} \leq C\|\nabla u\|_{L^{\mathcal{A}}\left(\mathbb{R}^{n}\right)}
$$

for every generalized Young function $\mathcal{A}$ with prescribed $\mathcal{A}_{\star}$ and for every $u \in W_{0}^{1, \mathcal{A}}\left(\mathbb{R}^{n}\right)$. Then $\mathcal{A}_{\star}$ satisfies (1.30). 
Proof. Part I. By Proposition 2.5, inequality (2.36) implies that (2.33) holds with $|G|=\infty$. On choosing test functions $f$ which vanish outside $[1, \infty)$, we have

$$
\begin{aligned}
\|f\|_{L^{A}(0, \infty)} & =\|f\|_{L^{A}(1, \infty)} \text { and } \\
\left\|\int_{s}^{\infty} r^{-1 / n^{\prime}} f(r) d r\right\|_{\bar{X}(0, \infty)} & \geq \int_{1}^{\infty} r^{-1 / n^{\prime}} f(r) d r\left\|\chi_{[0,1)}\right\|_{\bar{X}(0, \infty)} .
\end{aligned}
$$

From (2.33), (2.38) and (2.16) one gets

$$
\frac{K_{2}}{\left\|\chi_{[0,1)}\right\|_{\bar{X}(0, \infty)}} \geq \sup _{f \in L^{A}(1, \infty)} \frac{\int_{1}^{\infty} r^{-1 / n^{\prime}} f(r) d r}{\|f\|_{L^{A}(1, \infty)}} \geq\left\|r^{-1 / n^{\prime}}\right\|_{L^{\tilde{A}}(1, \infty)} .
$$

Hence, $\left\|r^{-1 / n^{\prime}}\right\|_{L^{\tilde{A}}(1, \infty)}<\infty$. This is equivalent to $\int^{\infty} \frac{\widetilde{A}(r)}{r^{1+n^{\prime}}} d r<\infty$, which is in turn equivalent to (1.6), by Lemma 2.3. The proof of Part II is analogous.

\section{Theorems 1.1 and 1.2: proof of the embeddings}

The present section is devoted to the proof of inequalities (1.20) and (1.22) of Theorem 1.1, and of inequalities (1.32) and (1.34) of Theorem 1.2. Consider Theorem 1.1. By Proposition 2.5, Part I, the proof of (1.20) is reduced to showing that, for any $A$ satisfying (1.6), a constant $K_{1}$, depending only on $n$, exists such that

$$
\left\|s^{-1 / n} \int_{s}^{\infty} r^{-1 / n^{\prime}} f(r) d r\right\|_{L^{B} A, n(0, \infty)} \leq K_{1}\|f\|_{L^{A}(0, \infty)}
$$

for every $f \in L^{A}(0, \infty)$. The same proposition ensures that inequality (1.22) is a consequence of (3.1) and of the fact that, under the additional assumption (1.21), there also exists a constant $K_{2}$, depending only on $n$ and on $\int_{0}^{\infty} \frac{\widetilde{A}(t)}{t^{1+n^{\prime}}} d t$, such that

$$
\left\|\int_{s}^{\infty} r^{-1 / n^{\prime}} f(r) d r\right\|_{L^{\infty}(0, \infty)} \leq K_{2}\|f\|_{L^{A}(0, \infty)}
$$

for every $f \in L^{A}(0, \infty)$.

Inequality (3.2) is an easy consequence of (2.8) and (2.16). Indeed,

$$
\begin{aligned}
\left\|\int_{s}^{\infty} r^{-1 / n^{\prime}} f(r) d r\right\|_{L^{\infty}(0, \infty)} & \leq \int_{0}^{\infty} r^{-1 / n^{\prime}}|f(r)| d r \leq 2\left\|r^{-1 / n^{\prime}}\right\|_{L^{\widetilde{A}}(0, \infty)}\|f\|_{L^{A}(0, \infty)} \\
& =2\left(n^{\prime} \int_{0}^{\infty} \frac{\widetilde{A}(t)}{t^{1+n^{\prime}}} d t\right)^{1 / n^{\prime}}\|f\|_{L^{A}(0, \infty)}
\end{aligned}
$$

for every $f \in L^{A}(0, \infty)$. Notice that $\int_{0}^{\infty} \frac{\widetilde{A}(t)}{t^{1+n^{\prime}}} d t<\infty$ by assumptions (1.6) and (1.21), and by Lemma 2.3. 
Inequality (3.1) is more delicate, and relies on the following interpolation theorem, of possible independent interest.

Theorem 3.1 Let $q \in(1, \infty)$ and let $A$ be a Young function satisfying (1.17). Then

$$
L^{A}(0, \infty) \subset L^{1}(0, \infty)+L^{q, 1}(0, \infty) .
$$

Moreover, let $T$ be any linear operator which is bounded from $L^{1}(0, \infty)$ into $L^{1}(0, \infty)$ with norm $\leq N_{1}$, and from $L^{q, 1}(0, \infty)$ into $L^{q, \infty}(0, \infty)$ with norm $\leq N_{q}$. Then $T$ is bounded from $L^{A}(0, \infty)$ into $L^{B_{A, q}}(0, \infty)$, and a constant $C$, depending only on $q$, exists such that

$$
\|T f\|_{L^{B_{A, q}(0, \infty)}} \leq C \max \left\{N_{1}, N_{q}\right\}\|f\|_{L^{A}(0, \infty)}
$$

for every $f \in L^{A}(0, \infty)$.

Remark 3.1 Theorem 3.1 can be transposed, with a completely analogous proof, to the more general setting of operators acting between function spaces defined on non-atomic $\sigma$-finite measure spaces. Furthermore, quasilinear operators, namely operators satisfying the inequality $|T(f+g)| \leq$ $k(|T f|+|T g|)$ for some $k>0$ and for every $f$ and $g$ can be allowed; in this case, the constant on the right-hand side of (3.4) has to be replaced by $k C \max \left\{N_{1}, N_{q}\right\}$.

In order to prove (3.1), we apply Theorem 3.1 to the linear operator $T$ defined as

$$
T f(s)=s^{-1 / n} \int_{s}^{\infty} r^{-1 / n^{\prime}} f(r) d r \quad \text { for } s>0,
$$

at any measurable function $f$ in $(0, \infty)$ which makes the integral on the right-hand side of (3.5) finite. The operator $T$ is bounded from $L^{1}(0, \infty)$ into $L^{1}(0, \infty)$ with norm $\leq n^{\prime}$, since

$$
\begin{aligned}
\|T f\|_{L^{1}(0, \infty)} & \leq \int_{0}^{\infty} s^{-1 / n} \int_{s}^{\infty} r^{-1 / n^{\prime}}|f(r)| d r d s \\
& =\int_{0}^{\infty} r^{-1 / n^{\prime}}|f(r)| \int_{0}^{r} s^{-1 / n} d s d r=n^{\prime}\|f\|_{L^{1}(0, \infty)} .
\end{aligned}
$$

Moreover, $T$ is bounded from $L^{n, 1}(0, \infty)$ into $L^{n, \infty}(0, \infty)$ with norm $\leq n$, since

$$
\begin{aligned}
\|T f\|_{L^{n, \infty}(0, \infty)} & =\sup _{s>0} s^{1 / n}(T f)^{*}(s) \leq \int_{0}^{\infty} r^{-1 / n^{\prime}}|f(r)| d r \\
& \leq \int_{0}^{\infty} r^{-1 / n^{\prime}} f^{*}(r) d r=\|f\|_{L^{n, 1}(0, \infty)} .
\end{aligned}
$$

Thus, (3.1) follows from Theorem 3.1. 
The proof of inequalities (1.32) and (1.34) of Theorem 1.2 makes use of Part II of Proposition 2.5 and is analogous.

Remark 3.2 It is clear from the above proof that inequality (3.1) continues to hold if $n$ is replaced by any $q \in(1, \infty)$ and $A$ is any Young function satisfying (1.17); the same is true for inequality (3.2) when $A$ fulfills

$$
\int^{\infty}\left(\frac{r}{A(r)}\right)^{1 /(q-1)} d r<\infty
$$

Proof of Theorem 3.1. In order to prove inclusion (3.3), we show that if $f$ is any measurable function in $(0, \infty)$ satisfying

$$
\int_{0}^{\infty} A(|f(s)|) d s<\infty
$$

and, for $t>0$, we decompose $f$ as $f=f_{t}+f^{t}$, where

$$
f_{t}(s)=\operatorname{sign}(f(s)) \min \{t,|f(s)|\} \text { and } f^{t}(s)=f(s)-f_{t}(s) \text { for } s>0
$$

then $f_{t} \in L^{q, 1}(0, \infty)$ and $f^{t} \in L^{1}(0, \infty)$. Owing to (1.17), we have $a(t)>0$ for $t>0$. Thus,

$$
\begin{aligned}
\left\|f^{t}\right\|_{L^{1}(0, \infty)} & =\int_{t}^{\infty}|\{|f|>\tau\}| d \tau \leq \frac{1}{a(t)} \int_{0}^{\infty}|\{|f|>\tau\}| a(\tau) d \tau \\
& =\frac{1}{a(t)} \int_{0}^{\infty} A(|f(s)|) d s<\infty
\end{aligned}
$$

On the other hand, $\int_{0}\left(\frac{1}{a(\tau)}\right)^{\frac{1}{q-1}} d \tau<\infty$, by assumption (1.17) and Lemma 2.3. Hence,

$$
\begin{aligned}
\left\|f_{t}\right\|_{L^{q, 1}(0, \infty)} & =\int_{0}^{t}|\{|f|>\tau\}|^{1 / q} d \tau \\
& \leq\left(\int_{0}^{\infty}|\{|f|>\tau\}| a(\tau) d \tau\right)^{1 / q}\left(\int_{0}^{t}\left(\frac{1}{a(\tau)}\right)^{\frac{1}{q-1}} d \tau\right)^{1 / q^{\prime}} \\
& =\left(\int_{0}^{t}\left(\frac{1}{a(\tau)}\right)^{\frac{1}{q-1}} d \tau\right)^{1 / q^{\prime}} \int_{0}^{\infty} A(|f(s)|) d s<\infty
\end{aligned}
$$

Inclusion (3.3) is proved. Such an inclusion ensures that the domain of $T$ contains the set of functions $f$ satisfying (3.6), and hence $T$ is well defined on $L^{A}(0, \infty)$. 
Let us now establish inequality (3.4). Throughout the proof, we shall denote the function $B_{A, q}$ simply by $B$. Assume that $f$ satisfies (3.6) and let $K$ be a positive constant to be chosen later. We have

$$
\begin{aligned}
\int_{0}^{\infty} B\left(\frac{T f(r)}{4 K}\right) d r= & \int_{0}^{\infty} b(t)|\{|T f|>4 K t\}| d t \\
\leq & \int_{0}^{\infty} \frac{B(2 t)}{t}|\{|T f|>4 K t\}| d t \\
= & \int_{0}^{\infty} \frac{B(t)}{t} \frac{d}{d t}\left(-\int_{t}^{\infty}|\{|T f|>2 K \tau\}| d \tau\right) d t \\
= & \left.\left(-\frac{B(t)}{t} \int_{t}^{\infty}|\{|T f|>2 K \tau\}| d \tau\right)\right|_{t=0} ^{t=\infty} \\
& +\int_{0}^{\infty} \frac{d}{d t}\left(\frac{B(t)}{t}\right)\left(\int_{t}^{\infty}|\{|T f|>2 K \tau\}| d \tau\right) \\
\leq & \limsup _{t \rightarrow 0+} \frac{B(t)}{t} \int_{t}^{\infty}|\{|T f|>2 K \tau\}| d \tau \\
& +\int_{0}^{\infty} \frac{d}{d t}\left(\frac{B(t)}{t}\right)\left(\int_{t}^{\infty}|\{|T f|>2 K \tau\}| d \tau\right) d t .
\end{aligned}
$$

Here, we have made use of the fact that $\int_{t}^{\infty}|\{|T f|>2 K \tau\}| d \tau<\infty$ for $t>0$, a consequence of estimates (3.14) and (3.19) below. We have also exploited the local absolute continuity of $B$, a finite-valued Young function. Now, let $\sigma:[0, \infty) \rightarrow[0, \infty]$ be the (non-decreasing) function given by $(2.25)$, and decompose $f$ as $f=f_{\sigma(t)}+f^{\sigma(t)}$, where $f_{\sigma(t)}$ and $f^{\sigma(t)}$ are defined as in (3.7), with $t$ replaced by $\sigma(t)$. By the linearity of $T$,

$$
|\{|T f|>2 K \tau\}| \leq\left|\left\{\left|T f_{\sigma(t)}\right|>K \tau\right\}\right|+\left|\left\{\left|T f^{\sigma(t)}\right|>K \tau\right\}\right| \text { for } t, \tau>0 .
$$

Inequalities (3.10) and (3.11) yield

$$
\begin{aligned}
& \int_{0}^{\infty} B\left(\frac{|T f(r)|}{4 K}\right) d r \leq \\
& \leq \limsup _{t \rightarrow 0+} \frac{B(t)}{t} \int_{t}^{\infty}\left|\left\{\left|T f_{\sigma(t)}\right|>K \tau\right\}\right| d \tau \\
&+\limsup _{t \rightarrow 0+} \frac{B(t)}{t} \int_{t}^{\infty}\left|\left\{\left|T f^{\sigma(t)}\right|>K \tau\right\}\right| d \tau \\
&+\int_{0}^{\infty} \frac{d}{d t}\left(\frac{B(t)}{t}\right)\left(\int_{t}^{\infty}\left|\left\{\left|T f_{\sigma(t)}\right|>K \tau\right\}\right| d \tau\right) d t \\
&+\int_{0}^{\infty} \frac{d}{d t}\left(\frac{B(t)}{t}\right)\left(\int_{t}^{\infty}\left|\left\{\left|T f^{\sigma(t)}\right|>K \tau\right\}\right| d \tau\right) d t
\end{aligned}
$$


Let us estimate the four summands on the right-hand side of (3.12). The boundedness of $T$ from $L^{q, 1}(0, \infty)$ into $L^{q, \infty}(0, \infty)$ comes into play in dealing with the terms involving $f_{\sigma(t)}$. By $(2.17)$, this property of $T$ implies that

$$
\left|\left\{\left|T f_{\sigma(t)}\right|>K \tau\right\}\right| \leq\left(\frac{N_{q}}{K \tau}\right)^{q}\left(\int_{0}^{\sigma(t)}|\{|f|>\theta\}|^{1 / q} d \theta\right)^{q} \text { for } t, \tau>0
$$

Hence, if $t>0$,

$$
\begin{aligned}
& \frac{B(t)}{t} \int_{t}^{\infty}\left|\left\{\left|T f_{\sigma(t)}\right|>K \tau\right\}\right| d \tau \leq \\
& \leq b(t)\left(\frac{N_{q}}{K}\right)^{q} \int_{t}^{\infty} \frac{1}{\tau^{q}}\left(\int_{0}^{\sigma(t)}|\{|f|>\theta\}|^{1 / q} d \theta\right)^{q} d \tau \\
& =\left(\frac{N_{q}}{K}\right)^{q} \frac{b(t)}{(q-1) t^{q-1}}\left(\int_{0}^{\sigma(t)}|\{|f|>\theta\}|^{1 / q} d \theta\right)^{q} \\
& \leq\left(\frac{N_{q}}{K}\right)^{q} \frac{b(t)}{(q-1) t^{q-1}}\left(\int_{0}^{\sigma(t)}\left(\frac{1}{a(\theta)}\right)^{\frac{1}{q-1}} d \theta\right)^{q-1} \int_{0}^{\infty}|\{|f|>\theta\}| a(\theta) d \theta \\
& \leq \frac{1}{(q-1)^{2}}\left(\frac{N_{q}}{K}\right)^{q} \int_{0}^{\infty} A(|f(s)|) d s,
\end{aligned}
$$

where the last inequality is due to (2.27), Lemma 2.4. Next, by (3.13) again,

$$
\begin{aligned}
\int_{0}^{\infty} \frac{d}{d t}\left(\frac{B(t)}{t}\right)\left(\int_{t}^{\infty}\left|\left\{\left|T f_{\sigma(t)}\right|>K \tau\right\}\right| d \tau\right) d t \\
\leq\left(\frac{N_{q}}{K}\right)^{q} \int_{0}^{\infty} \frac{d}{d t}\left(\frac{B(t)}{t}\right)\left(\int_{t}^{\infty} \frac{1}{\tau^{q}}\left(\int_{0}^{\sigma(t)}|\{|f|>\theta\}|^{1 / q} d \theta\right)^{q} d \tau\right) d t \\
\quad=\left(\frac{N_{q}}{K}\right)^{q} \int_{0}^{\infty}\left(\int_{t}^{\infty} \tau^{-q} d \tau\right) \frac{d}{d t}\left(\frac{B(t)}{t}\right)\left(\int_{0}^{\sigma(t)}|\{|f|>\theta\}|^{1 / q} d \theta\right)^{q} d t \\
\quad=\left(\frac{N_{q}}{K}\right)^{q} \int_{0}^{\infty} \tau^{-q}\left(\int_{0}^{\tau} \frac{d}{d t}\left(\frac{B(t)}{t}\right)\left(\int_{0}^{\sigma(t)}|\{|f|>\theta\}|^{1 / q} d \theta\right)^{q} d t\right) d \tau \\
\leq\left(\frac{N_{q}}{K}\right)^{q} \int_{0}^{\infty} \tau^{-q}\left(\int_{0}^{\sigma(\tau)}|\{|f|>\theta\}|^{1 / q} d \theta\right)^{q}\left(\int_{0}^{\tau} \frac{d}{d t}\left(\frac{B(t)}{t}\right) d t\right) d \tau \\
\leq\left(\frac{N_{q}}{K}\right)^{q} \int_{0}^{\infty} \frac{b(\tau)}{\tau^{q}}\left(\int_{0}^{\sigma(\tau)}|\{|f|>\theta\}|^{1 / q} d \theta\right)^{q} d \tau .
\end{aligned}
$$

A generalized version of the weighted Hardy inequality (see [12, Lemma 1]) tells us that (2.26) is a sufficient (and necessary, up to the constant) condition for the inequality

$$
\int_{0}^{\infty} \frac{b(\tau)}{\tau^{q}}\left(\int_{0}^{\sigma(\tau)} h(\theta) d \theta\right)^{q} d \tau \leq\left[\frac{\left(q^{\prime}\right)^{1 / q^{\prime}} q^{1 / q}}{(q-1)^{2 / q}}\right]^{q} \int_{0}^{\infty} a(\tau) h(\tau)^{q} d \tau
$$

to hold for every measurable function $h:[0, \infty) \rightarrow[0, \infty)$. 
On applying (3.16) with $h(t)=|\{|f|>t\}|^{1 / q}$, we infer from (3.15) that

$$
\begin{aligned}
\int_{0}^{\infty} \frac{d}{d t}\left(\frac{B(t)}{t}\right) & \left(\int_{t}^{\infty}\left|\left\{\left|T f_{\sigma(t)}\right|>K \tau\right\}\right| d \tau\right) d t \leq \\
& \leq\left(\frac{N_{q}}{K}\right)^{q} \frac{q^{q}}{(q-1)^{q+1}} \int_{0}^{\infty} a(t)|\{|f|>t\}| d t \\
& =\left(\frac{N_{q}}{K}\right)^{q} \frac{q^{q}}{(q-1)^{q+1}} \int_{0}^{\infty} A(|f(s)|) d s .
\end{aligned}
$$

The boundedness of $T$ from $L^{1}(0, \infty)$ into $L^{1}(0, \infty)$ plays its role in the estimate of the summands depending on $f^{\sigma(t)}$ on the right-hand side of (3.12). Such an assumption on $T$ ensures that

$$
\int_{t}^{\infty}\left|\left\{\left|T f^{\sigma(t)}\right|>K \tau\right\}\right| d \tau \leq \frac{N_{1}}{K} \int_{\sigma(t)}^{\infty}|\{|f|>\tau\}| d \tau \quad \text { for } t>0 .
$$

Thus, by (2.25),

$$
\begin{aligned}
\frac{B(t)}{t} \int_{t}^{\infty} \mid\left\{\left|T f^{\sigma(t)}\right|\right. & >K \tau\}\left|d \tau \leq \frac{N_{1}}{K} b(t) \int_{\sigma(t)}^{\infty}\right|\{|f|>\tau\} \mid d \tau \\
& \leq \frac{N_{1}}{K} \frac{b(t)}{a(\sigma(t))} \int_{\sigma(t)}^{\infty} a(\tau)|\{|f|>\tau\}| d \tau \\
& \leq \frac{N_{1}}{K} \int_{0}^{\infty} a(\tau)|\{|f|>\tau\}| d \tau=\frac{N_{1}}{K} \int_{0}^{\infty} A(|f(s)|) d s .
\end{aligned}
$$

Inequality (3.18) and equation (2.25) also imply that

$$
\begin{aligned}
\int_{0}^{\infty} \frac{d}{d t}\left(\frac{B(t)}{t}\right) & \left(\int_{t}^{\infty}\left|\left\{\left|T f^{\sigma(t)}\right|>K \tau\right\}\right| d \tau\right) d t \leq \\
& \leq \frac{N_{1}}{K} \int_{0}^{\infty} \frac{d}{d t}\left(\frac{B(t)}{t}\right)\left(\int_{\sigma(t)}^{\infty}|\{|f|>\tau\}| d \tau\right) d t \\
& =\frac{N_{1}}{K} \int_{0}^{\infty}\left(\int_{0}^{\sigma^{-1}(\tau)} \frac{d}{d t}\left(\frac{B(t)}{t}\right) d t\right)|\{|f|>\tau\}| d \tau \\
& \leq \frac{N_{1}}{K} \int_{0}^{\infty} b\left(\sigma^{-1}(\tau)\right)|\{|f|>\tau\}| d \tau \\
& \leq \frac{N_{1}}{K} \int_{0}^{\infty} a(\tau)|\{|f|>\tau\}| d \tau=\frac{N_{1}}{K} \int_{0}^{\infty} A(|f(s)|) d s
\end{aligned}
$$

where $\sigma^{-1}$ is the (generalized) left-continuous inverse of $\sigma$.

Combining (3.12), (3.14), (3.17), (3.19) and (3.20) leads to

$$
\int_{0}^{\infty} B\left(\frac{|T f(r)|}{4 K}\right) d r \leq\left[\left(\frac{N_{q}}{K}\right)^{q} \frac{(q-1)^{q-1}+q^{q}}{(q-1)^{q+1}}+\frac{2 N_{1}}{K}\right] \int_{0}^{\infty} A(|f(s)|) d s
$$


The choice $K=\max \left\{N_{1}, N_{2}\right\}$ in (3.21) yields

$$
\int_{0}^{\infty} B\left(\frac{|T f(r)|}{4 C \max \left\{N_{1}, N_{2}\right\}}\right) d r \leq \int_{0}^{\infty} A(|f(s)|) d s
$$

with $C=\frac{(q-1)^{q-1}+q^{q}}{(q-1)^{q+1}}+2$. In the derivation of (3.22) one has to make use of (2.12) and of the fact that $C>1$. Inequality (3.4) follows from (3.22), by the very definition of Luxemburg norm.

\section{Theorems 1.1 and 1.2: optimality}

Our task in this section is to prove the second part of the statements of Theorems 1.1 and 1.2, where it is claimed that the spaces appearing on the right-hand sides of inequalities (1.20), (1.22) and (1.32), (1.34) are the best possible among all r.i. spaces. Notice that we only need to consider Theorem 1.1, since the result in Theorem 1.2 follows on choosing $\mathcal{A}(\xi)=$ $\mathcal{A}_{\star}(|\xi|)$ and applying Theorem 1.1.

Thanks to Proposition 2.5, the question of the optimality of the spaces $L\left(n, B_{A, n}\right)(G)$ and $L\left(n, B_{A, n}\right)(G) \cap L^{\infty}(G)$ in (1.20) and (1.22), respectively, is equivalent to a corresponding problem for a one-dimensional inequality. The crucial step in attacking this problem turns out to be the Hölder type inequality for non-increasing functions in Orlicz spaces contained in the following theorem.

Theorem 4.1 Let $q \in(1, \infty)$ and let $A$ be a Young function satisfying (1.17).

I. Assume that

$$
\int^{\infty}\left(\frac{r}{A(r)}\right)^{\frac{1}{q-1}} d r=\infty .
$$

Then there exists a constant $K_{1}(q)$, depending only on $q$, such that

$$
\int_{0}^{\infty}|f(s) g(s)| d s \leq K_{1}(q)\left\|s^{-1 / q} f^{*}(s)\right\|_{L^{B A, q}(0, \infty)}\left\|s^{1 / q} g^{* *}(s)\right\|_{L^{\tilde{A}}(0, \infty)}
$$

for any measurable functions $f$ and $g$ in $(0, \infty)$.

II. Assume that

$$
\int^{\infty}\left(\frac{r}{A(r)}\right)^{\frac{1}{q-1}} d r<\infty .
$$

Then a constant $\alpha(A, q)$, depending only on $A$ and $q$, exists such that inequality (4.2) holds for every measurable function $g$ in $(0, \infty)$ and every measurable function $f$ in $(0, \infty)$ satisfying

$$
|\{s \in(0, \infty):|f(s)|=\operatorname{ess} \sup |f|\}| \geq \alpha(q, A) .
$$


Remark 4.1 Obviously, owing to the Hardy-Littlewood inequality (2.5), a completely analogous version of Theorem 4.1 holds if $f$ and $g$ are replaced by functions $u$ and $v$ defined on a measurable set $G \subseteq \mathbb{R}^{n}$.

The proof of Theorem 4.1 is split into several lemmas and is presented in the second part of this section.

We first accomplish the proof of Theorem 1.1. Let us begin with Part I, and let us assume that (1.19) is fulfilled. Consider the case where $G=\mathbb{R}^{n}$. We have to show that, if $X\left(\mathbb{R}^{n}\right)$ is an r.i. space satisfying (2.32) with $G=\mathbb{R}^{n}$, then a constant $K$ exists such that

$$
\|u\|_{X\left(\mathbb{R}^{n}\right)} \leq K\|u\|_{L\left(n, B_{A, n}\right)\left(\mathbb{R}^{n}\right)}
$$

for every $u \in L\left(n, B_{A, n}\right)\left(\mathbb{R}^{n}\right)$. By Proposition 2.5, inequality (2.33) holds with $|G|=\infty$. We now make use of a duality argument, involving associate spaces (see also [10, Proof of Lemma 1] and [18, Proof of Theorem 4.6]). By (2.33), (2.6) and (2.16),

$$
\begin{aligned}
& K_{2} \geq \sup _{\substack{f \in L^{A}(0, \infty) \\
f \geq 0}} \frac{\left\|\int_{s}^{\infty} r^{-1 / n^{\prime}} f(r) d r\right\|_{\bar{X}(0, \infty)}}{\|f\|_{L^{A}(0, \infty)}} \\
& =\sup _{\substack{f \in L^{A}(0, \infty) \\
f \geq 0}} \sup _{g \in \bar{X}^{\prime}(0, \infty)} \frac{\int_{0}^{\infty} g^{*}(s) \int_{s}^{\infty} r^{-1 / n^{\prime}} f(r) d r d s}{\|g\|_{\bar{X}^{\prime}(0, \infty)}\|f\|_{L^{A}(0, \infty)}} \\
& =\sup _{g \in \bar{X}^{\prime}(0, \infty)} \sup _{\substack{f \in L^{A}(0, \infty) \\
f \geq 0}} \frac{\int_{0}^{\infty} f(r) r^{-1 / n^{\prime}} \int_{0}^{r} g^{*}(s) d s d r}{\|g\|_{\bar{X}^{\prime}(0, \infty)}\|f\|_{L^{A}(0, \infty)}} \\
& \geq \sup _{g \in \bar{X}^{\prime}(0, \infty)} \frac{\left\|r^{-1 / n^{\prime}} \int_{0}^{r} g^{*}(s) d s\right\|_{L^{\tilde{A}}(0, \infty)}}{\|g\|_{\bar{X}^{\prime}(0, \infty)}} .
\end{aligned}
$$

Hence,

$$
\left\|r^{-1 / n^{\prime}} \int_{0}^{r} g^{*}(s) d s\right\|_{L^{\tilde{A}}(0, \infty)} \leq K_{2}\|g\|_{\bar{X}^{\prime}(0, \infty)}
$$

for every $g \in \bar{X}^{\prime}(0, \infty)$. From inequality (4.7) and from Theorem 4.1, Part I, we infer that

$$
\begin{aligned}
& \sup _{g \in \bar{X}^{\prime}(0, \infty)} \frac{\int_{0}^{\infty} f^{*}(s) g^{*}(s) d s}{\|g\|_{\bar{X}^{\prime}(0, \infty)}} \leq K_{2} \sup _{g \in \bar{X}^{\prime}(0, \infty)} \frac{\int_{0}^{\infty} f^{*}(s) g^{*}(s) d s}{\left\|r^{-1 / n^{\prime}} \int_{0}^{r} g^{*}(s) d s\right\|_{L^{\tilde{A}}(0, \infty)}} \\
& \quad \leq K_{2} K_{1}(n)\left\|s^{-1 / n} f^{*}(s)\right\|_{L^{B, n}(0, \infty)}=K_{2} K_{1}(n)\|f\|_{L\left(n, B_{A, n}\right)(0, \infty)}
\end{aligned}
$$

for every $f \in L\left(n, B_{A, n}\right)(0, \infty)$. 
By (2.7) and (2.9), the first supremum in (4.8) equals $\|f\|_{\bar{X}(0, \infty)}$. Thus, we conclude that

$$
\|f\|_{\bar{X}(0, \infty)} \leq K_{2} K_{1}(n)\|f\|_{L\left(n, B_{A, n}\right)(0, \infty)}
$$

for every $f \in L\left(n, B_{A, n}\right)(0, \infty)$, whence (4.5) follows, owing to (2.9).

Let us now take into account the case where $G$ is an open subset of $\mathbb{R}^{n}$ having finite measure. Let $X(G)$ be any r.i. space satisfying (2.32). Via the same argument as above, one deduces that

$$
\left\|r^{-1 / n^{\prime}} \int_{0}^{r} g^{*}(s) d s\right\|_{L^{\tilde{A}}(0,|G|)} \leq K_{2}\|g\|_{\bar{X}^{\prime}(0,|G|)}
$$

for every $g \in \bar{X}^{\prime}(0,|G|)$. Theorem 4.1 implies that

$$
\int_{0}^{|G|} f^{*}(s) g^{*}(s) d s \leq K_{1}(n)\left\|s^{-1 / n} f^{*}(s)\right\|_{L^{B_{A}, n}(0,|G|)}\left\|s^{-1 / n^{\prime}} \int_{0}^{s} g^{*}(r) d r\right\|_{L^{\tilde{A}}(0, \infty)}
$$

for any measurable functions $f$ and $g$ in $(0, \infty)$, vanishing outside $(0,|G|)$. We claim that a constant $C$, depending only $A,|G|$ and $n$, exists such that

$$
\left\|s^{-1 / n^{\prime}} \int_{0}^{s} g^{*}(r) d r\right\|_{L^{\tilde{A}(0, \infty)}} \leq C\left\|s^{-1 / n^{\prime}} \int_{0}^{s} g^{*}(r) d r\right\|_{L^{\tilde{A}(0,|G|)}}
$$

for every $g$ vanishing outside $(0,|G|)$. Indeed, we have

$$
\begin{aligned}
\left\|s^{-1 / n^{\prime}} \int_{0}^{s} g^{*}(r) d r\right\|_{L^{\tilde{A}}(0, \infty)} \leq & \left\|s^{-1 / n^{\prime}} \int_{0}^{s} g^{*}(r) d r\right\|_{L^{\tilde{A}}(0,|G|)} \\
& +\left(\int_{0}^{|G|} g^{*}(r) d r\right)\left\|s^{-1 / n^{\prime}} \chi_{(|G|, \infty)}(s)\right\|_{L^{\tilde{A}}(0, \infty)}
\end{aligned}
$$

Notice that the last norm is finite since $\int_{0} \frac{\widetilde{A}(t)}{t^{1+n^{\prime}}} d t<\infty$, as a consequence of (1.6) and of Lemma 2.3. Now,

$$
\begin{aligned}
& \frac{n|G|^{1 / n}}{n+1} \int_{0}^{|G|} g^{*}(r) d r=g^{* *}(|G|) \int_{0}^{|G|} s^{1 / n} d s \\
& \quad \leq \int_{0}^{|G|} s^{1 / n} g^{* *}(s) d s \leq 2\|1\|_{L^{A}(0,|G|)}\left\|s^{-1 / n^{\prime}} \int_{0}^{s} g^{*}(r) d r\right\|_{L^{\tilde{A}(0,|G|)}} .
\end{aligned}
$$

Combining (4.12)-(4.13) yields (4.11). On making use of (4.9)-(4.11), one can argue as in the case where $G=\mathbb{R}^{n}$ and conclude that there exists a constant $K$ such that $\|u\|_{X(G)} \leq K\|u\|_{L\left(n, B_{A, n}\right)(G)}$ for every $u \in L\left(n, B_{A, n}\right)(G)$. 
Consider Part II. The optimality of $L\left(n, B_{A, n}\right)(G) \cap L^{\infty}(G)$ is clear when $|G|<\infty$, since, in this case, $L\left(n, B_{A, n}\right)(G) \cap L^{\infty}(G)=L^{\infty}(G) \subseteq X(G)$ for any r.i. space $X(G)$, thanks to property $(\mathrm{P} 4)$.

Assume, instead, that $G=\mathbb{R}^{n}$. Owing to (2.10), it suffices to show that

$$
L\left(n, B_{A, n}\right)\left(\mathbb{R}^{n}\right) \cap L^{\infty}\left(\mathbb{R}^{n}\right) \subseteq X\left(\mathbb{R}^{n}\right)
$$

for every r.i. space $X\left(\mathbb{R}^{n}\right)$ satisfying (2.32) with $G=\mathbb{R}^{n}$.

Let $u \in L\left(n, B_{A, n}\right)\left(\mathbb{R}^{n}\right) \cap L^{\infty}\left(\mathbb{R}^{n}\right)$. Then, in particular, $u$ is essentially bounded. Thus, if $|\operatorname{supp} u|<\infty$, then $u$ belongs to any r.i. space, and hence to $u \in X\left(\mathbb{R}^{n}\right)$, by properties (P4)-(P5) of r.i. spaces. Assume now that $|\operatorname{supp} u|=\infty$. Given $t>0$, let us decompose $u$ as $u(x)=u_{t}(x)+u^{t}(x)$ for $x \in \mathbb{R}^{n}$, where $u_{t}$ and $u^{t}$ are defined analogously as in (3.7). Notice that $u_{t}$ and $u^{t}$ belong to $L\left(n, B_{A, n}\right)\left(\mathbb{R}^{n}\right) \cap L^{\infty}\left(\mathbb{R}^{n}\right)$, since $u$ does and $\left|u_{t}(x)\right| \leq|u(x)|$, $\left|u^{t}(x)\right| \leq|u(x)|$ for $x \in \mathbb{R}^{n}$. We have $\left|\operatorname{supp} u^{t}\right|=|\{|u|>t\}|<\infty$, since $u \in L\left(n, B_{A, n}\right)\left(\mathbb{R}^{n}\right)$. Inasmuch as $u^{t}$ is essentially bounded, we conclude as above that $u^{t} \in X\left(\mathbb{R}^{n}\right)$ for every $t>0$. Next, consider $u_{t}$. Since we are assuming that $|\operatorname{supp} u|=\infty$, then $\left|\left\{u_{t} \geq t\right\}\right|=|\{|u| \geq t\}| \geq \alpha(q, A)$, provided that $t$ is sufficiently small, where $\alpha(q, A)$ is the number appearing in Theorem 4.1. Thus, since ess $\sup \left|u_{t}\right|=t$, then the function $f=u_{t}^{*}$ satisfies assumption (4.4) of Theorem 4.1. The same argument as in the proof of Part I now yields $u_{t} \in X\left(\mathbb{R}^{n}\right)$. Hence, $u \in X\left(\mathbb{R}^{n}\right)$. The proof of Theorem 1.1 is complete.

Let us now come to Theorem 4.1. The outline of the proof is as follows. Inequality (4.2) is first transformed into an equivalent inequality where the product of norms is replaced by a sum of integrals on the right-hand side. Via a discretization and truncation argument, this inequality is further reduced to an inequality restricted to characteristic functions of intervals and linear combinations of them, which is dealt with in Lemma 4.4. The proof of this lemma requires an inequality between certain Orlicz norms of powers (Lemma 4.3), which in turn relies upon an inequality involving integrals of the function $A$ (Lemma 4.2).

As a preliminary step, we associate with any $A$ and $q$ as in the statement of Theorem 4.1 the functions $\Lambda, \Phi, \Psi, \Theta:[0, \infty] \rightarrow[0, \infty]$ defined, for $s \geq 0$, by:

$$
\Lambda(s)=\int_{0}^{s}\left(\frac{1}{a(r)}\right)^{\frac{1}{q-1}} d r
$$

where $a$ is the function appearing in (1.4),

$$
\Phi(s)=q^{\prime} \int_{0}^{s} \frac{\widetilde{A}(r)}{r^{1+q^{\prime}}} d r,
$$




$$
\Psi(s)=\left(s \Phi^{-1}\left(s^{q^{\prime}}\right)\right)^{q^{\prime}},
$$

where $\Phi^{-1}$ is the (generalized) right-continuous inverse of $\Phi$, and

$$
\Theta(s)=\left(\int_{s / q^{\prime}}^{\infty}\left(\frac{1}{b^{-1}(r)}\right)^{q-1} d r\right)^{\frac{1}{1-q}}
$$

where $b^{-1}$ is given by $(1.18)$.

Lemma 4.2 Let $q \in(1, \infty)$ and let $A$ be a Young function satisfying (1.17). Let $\Phi$ and $\Theta$ be the functions defined by (4.16) and (4.18). Then there exists a constant $K_{2}(q)$, depending only on $q$, such that

$$
\Theta\left(\frac{s}{\left(K_{2}(q)\right)^{q^{\prime}}}\right) \leq\left(K_{2}(q)\right)^{q^{\prime}} \Phi(s) \quad \text { for } s \in(0, \beta(A, q)),
$$

where

$$
\beta(A, q)= \begin{cases}a\left(\Lambda^{-1}\left(2^{\frac{1}{1-q}} \Lambda(\infty)\right)\right) & \text { if } \int^{\infty}\left(\frac{1}{a(r)}\right)^{1 /(q-1)} d r<\infty \\ \infty & \text { if } \int^{\infty}\left(\frac{1}{a(r)}\right)^{1 /(q-1)} d r=\infty\end{cases}
$$

and $\Lambda$ is given by (4.15)

Proof. Inequality (4.19) reads

$$
\left(\int_{s /\left(q^{\prime}\left(K_{2}(q)\right)^{\prime}\right)}^{\infty}\left(\frac{1}{b^{-1}(r)}\right)^{q-1} d r\right)^{\frac{1}{1-q}} \leq q^{\prime}\left(K_{2}(q)\right)^{q^{\prime}} \int_{0}^{s} \frac{\widetilde{A}(r)}{r^{1+q^{\prime}}} d r
$$

Consider the left-hand side of (4.21). We have, by Fubini's theorem,

$$
\begin{aligned}
(q-1) \int_{s}^{\infty}\left(\frac{1}{b^{-1}(r)}\right)^{q-1} d r= & (q-1) \int_{s}^{\infty}\left(\int_{a^{-1}(r)}^{\infty}\left(\int_{0}^{t}\left(\frac{1}{a(\tau)}\right)^{\frac{1}{q-1}} d \tau\right)^{-q} \frac{d t}{a(t)^{q^{\prime}}}\right) d r \\
= & (q-1) \int_{a^{-1}(s)}^{\infty}\left(\int_{0}^{t}\left(\frac{1}{a(\tau)}\right)^{\frac{1}{q-1}} d \tau\right)^{-q} \frac{a(t)-s}{a(t)^{q^{\prime}}} d t \\
= & \left(\int_{0}^{a^{-1}(s)}\left(\frac{1}{a(\tau)}\right)^{\frac{1}{q-1}} d \tau\right)^{1-q}-\left(\int_{0}^{\infty}\left(\frac{1}{a(\tau)}\right)^{\frac{1}{q-1}} d \tau\right)^{1-q} \\
& \quad-s \int_{a^{-1}(s)}^{\infty}\left(\int_{0}^{t}\left(\frac{1}{a(\tau)}\right)^{\frac{1}{q-1}} d \tau\right)^{-q} \frac{d t}{a(t)^{q^{\prime}}} \quad \text { for } s>0 . \\
(4.22) \quad & \left(\Lambda\left(a^{-1}(s)\right)\right)^{1-q}-(\Lambda(\infty))^{1-q}-\frac{s}{\left(b^{-1}(s)\right)^{q-1}} \quad \text {. }
\end{aligned}
$$


Moreover,

$$
\int_{s / 2}^{\infty}\left(\frac{1}{b^{-1}(r)}\right)^{q-1} d r \geq \int_{s / 2}^{s}\left(\frac{1}{b^{-1}(r)}\right)^{q-1} d r \geq \frac{s}{2\left(b^{-1}(s)\right)^{q-1}} \quad \text { for } s>0
$$

Combining (4.22)-(4.23) yields

$$
\left[\left(\Lambda\left(a^{-1}(s)\right)\right)^{1-q}-(\Lambda(\infty))^{1-q}\right]^{\frac{1}{1-q}} \geq(q+1)^{\frac{1}{1-q}}\left(\int_{s / 2}^{\infty}\left(\frac{1}{b^{-1}(r)}\right)^{q-1} d r\right)^{\frac{1}{1-q}}
$$

for $s>0$. As far as the right-hand side of (4.21) is concerned, an application of Fubini's theorem gives

$$
\begin{aligned}
\int_{0}^{s} \frac{a^{-1}(\tau)}{\tau^{q^{\prime}}} d \tau & =\int_{0}^{a^{-1}(s)} \int_{a(r)}^{s} \tau^{-q^{\prime}} d \tau d r \\
& =(q-1)\left(\int_{0}^{a^{-1}(s)}\left(\frac{1}{a(r)}\right)^{\frac{1}{q-1}} d r-\frac{a^{-1}(s)}{s^{1 /(q-1)}}\right)
\end{aligned}
$$

for $s>0$. On the other hand,

$$
\int_{0}^{2 s} \frac{a^{-1}(\tau)}{\tau^{q^{\prime}}} d \tau \geq \int_{s}^{2 s} \frac{a^{-1}(\tau)}{\tau^{q^{\prime}}} d \tau \geq(q-1)\left(1-2^{1 /(1-q)}\right) \frac{a^{-1}(s)}{s^{1 /(q-1)}}
$$

for $s>0$. On making first use of (4.25)-(4.26), and then of (2.11) with $A$ replaced by $\widetilde{A}$ and $a$ replaced by $a^{-1}$, we easily deduce that

$$
\begin{aligned}
\Lambda\left(a^{-1}(s)\right) & =\int_{0}^{a^{-1}(s)}\left(\frac{1}{a(r)}\right)^{\frac{1}{q-1}} d r \leq \frac{2-2^{1 /(1-q)}}{(q-1)\left(1-2^{1 /(1-q)}\right)} \int_{0}^{2 s} \frac{a^{-1}(\tau)}{\tau^{q^{\prime}}} d \tau \\
(4.27) & \leq \frac{2^{q^{\prime}}\left(2-2^{1 /(1-q)}\right)}{(q-1)\left(1-2^{1 /(1-q)}\right)} \int_{0}^{4 s} \frac{\widetilde{A}(\tau)}{\tau^{1+q^{\prime}}} d \tau
\end{aligned}
$$

for $s>0$. Now, if $\int^{\infty}\left(\frac{r}{A(r)}\right)^{1 /(q-1)} d r=\infty$, then $\Lambda(\infty)^{1-q}=0$, by Lemma 2.3 . Thus, inequality (4.21) holds for every $s>0$, as a consequence of (4.24) and (4.27). If, on the contrary, $\int^{\infty}\left(\frac{r}{A(r)}\right)^{1 /(q-1)} d r<\infty$, then $\Lambda(\infty)^{1-q}>0$, and the very definition of $\beta(A, q)$ easily implies that

$$
\left[\left(\Lambda\left(a^{-1}(s)\right)\right)^{1-q}-(\Lambda(\infty))^{1-q}\right]^{\frac{1}{1-q}} \leq \frac{1}{2} \Lambda\left(a^{-1}(s)\right) \text { for } 0<s \leq \beta(A, q) .
$$

From (4.24), (4.27) and (4.28) we infer that (4.21) holds for $s \in(0, \beta(A, q)]$. 
Lemma 4.3 Let $q \in(1, \infty)$ and let $A$ be a Young function satisfying (1.17). Then

$$
1 \leq 2 K_{2}(q)\left\|s^{-1 / q} \chi_{[0, t)}(s)\right\|_{L^{B} A, q(0, \infty)}\left\|s^{-1 / q^{\prime}} \chi_{[t, \infty)}(s)\right\|_{L^{\tilde{A}}(0, \infty)}
$$

for every $t \geq \alpha(A, q) / 2$. Here

$$
\alpha(A, q)= \begin{cases}\frac{2}{\Psi\left(\frac{1}{K_{2}(q)} \Theta^{1 / q^{\prime}}\left(\frac{\beta(A, q)}{\left(K_{2}(q)\right)^{q^{\prime}}}\right)\right)} & \text { if } \int^{\infty}\left(\frac{r}{A(r)}\right)^{1 /(q-1)} d r<\infty \\ 0 & \text { if } \int^{\infty}\left(\frac{r}{A(r)}\right)^{1 /(q-1)} d r=\infty,\end{cases}
$$

where $\Psi$ and $\Theta$ are defined in (4.17) and (4.18), respectively, $K_{2}(q)$ is the constant appearing in (4.19) and $\beta(A, q)$ is defined by (4.20).

Proof. Computations show that

$$
\left\|s^{-1 / q} \chi_{[0, t)}(s)\right\|_{L^{B}{ }_{A, q}(0, \infty)}=\frac{t^{-1 / q}}{H^{-1}(1 / t)} \quad \text { for } t>0
$$

where $H^{-1}$ is the (generalized) right-continuous inverse of the Young function $H$ given by

$$
H(s)=q s^{q} \int_{s}^{\infty} \frac{B_{A, q}(r)}{r^{1+q}} d r \quad \text { for } s>0 .
$$

[13, Lemma 5] ensures that

$$
\widetilde{H}(s) \leq q^{\prime}\left(\frac{s}{q^{\prime}}\right)^{q^{\prime}}\left(\int_{s / q^{\prime}}^{\infty}\left(\frac{r}{\widetilde{B_{A, q}}(r)}\right)^{q-1} d r\right)^{\frac{1}{1-q}} \text { for } s>0 .
$$

Hence, owing to (2.11) applied to $B_{A, q}$, we have $\widetilde{H}(s) \leq s^{q^{\prime}} \Theta(s)$ for $s>0$. Thus, if $F:[0, \infty) \rightarrow[0, \infty]$ is defined as $F(s)=s^{q^{\prime}} \Theta(s)$ for $s \geq 0$, then

$$
\widetilde{H}^{-1}(r) \geq F^{-1}(r) \text { for } r>0,
$$

where $\widetilde{H}^{-1}$ and $F^{-1}$ are (generalized) right-continuous inverses. From (4.31), from (2.15) applied to $H$, and from (4.34) we deduce that

$$
\left\|s^{-1 / q} \chi_{[0, t)}(s)\right\|_{L^{B} A, q(0, \infty)} \geq \frac{F^{-1}(1 / t)}{2 t^{-1 / q^{\prime}}} \quad \text { for } t>0 .
$$

On the other hand, it is not difficult to verify that

$$
\left\|s^{-1 / q^{\prime}} \chi_{[t, \infty)}(s)\right\|_{L^{\tilde{A}}(0, \infty)}=\Psi^{-1}(1 / t) \text { for } t>0,
$$

where $\Psi^{-1}$ is the (generalized) right-continuous inverse of $\Psi$. 
Now, define $L:[0, \infty) \rightarrow[0, \infty]$ as $L(s)=\left(s \Theta^{-1}\left(s^{q^{\prime}}\right)\right)^{q^{\prime}}$ for $s>0$, and observe that

$$
L^{-1}(r)=\frac{r^{1 / q^{\prime}}}{F^{-1}(r)} \quad \text { for } r>0,
$$

where $L^{-1}$ is the (generalized) right-continuous inverse of $L$. Lemma 4.2 implies that

$$
\left(K_{2}(q)\right)^{q^{\prime}} \Theta^{-1}\left(\tau\left(K_{2}(q)\right)^{q^{\prime}}\right) \geq \Phi^{-1}(\tau) \text { for } \tau>0
$$

[respectively, for $\left.0<\tau \leq\left(K_{2}(q)\right)^{-q^{\prime}} \Theta\left(\beta(A, q)\left(K_{2}(q)\right)^{-q^{\prime}}\right)\right]$ if

$$
\int^{\infty}\left(\frac{r}{A(r)}\right)^{1 /(q-1)} d r=\infty \quad\left[\int^{\infty}\left(\frac{r}{A(r)}\right)^{1 /(q-1)} d r<\infty\right]
$$

Hence,

$$
L\left(K_{2}(q) s\right) \geq \Psi(s) \text { for } s>0 \quad\left[0<s \leq\left(K_{2}(q)\right)^{-1} \Theta^{1 / q^{\prime}}\left(\beta(A, q)\left(K_{2}(q)\right)^{-q^{\prime}}\right)\right] .
$$

Thus,

$$
L^{-1}(r) \leq K_{2}(q) \Psi^{-1}(r)
$$

for $r>0\left[0<r \leq \Psi\left(\left(K_{2}(q)\right)^{-1} \Theta^{1 / q^{\prime}}\left(\beta(A, q)\left(K_{2}(q)\right)^{-q^{\prime}}\right)\right)\right]$. Inequality (4.29) is a consequence of $(4.35)-(4.38)$.

Lemma 4.4 Let $q \in(1, \infty)$ and let $A$ be a Young function satisfying (1.17). Then

$$
\begin{aligned}
\int_{0}^{\infty} \lambda \chi_{[0, t)}(s) \sum_{i \in \mathbb{Z}} \lambda_{i} \chi_{\left[t_{i}, t_{i+1}\right)}(s) d s & \leq 2 K_{2}(q)\left[\int_{0}^{\infty} B_{A, q}\left(\lambda s^{-1 / q} \chi_{[0, t)}(s)\right) d s\right. \\
& \left.+\int_{0}^{\infty} \widetilde{A}\left(s^{-1 / q^{\prime}} \int_{0}^{s} \sum_{i \in \mathbb{Z}} \lambda_{i} \chi_{\left[t_{i}, t_{i+1}\right)}(r) d r\right) d s\right]
\end{aligned}
$$

for all nonnegative $\lambda, \lambda_{i}, i \in \mathbb{Z}$, for all nonnegative $t_{i}$ such that $t_{i} \leq t_{i+1}$ for $i \in \mathbb{Z}$, and for all

$$
\begin{aligned}
t \geq \frac{\alpha(A, q)}{2}\left[\int_{0}^{\infty} B_{A, q}\left(\lambda s^{-1 / q} \chi_{[0, t)}(s)\right) d s\right. \\
\left.\quad+\int_{0}^{\infty} \widetilde{A}\left(s^{-1 / q^{\prime}} \int_{0}^{s} \sum_{i \in \mathbb{Z}} \lambda_{i} \chi_{\left[t_{i}, t_{i+1}\right)}(r) d r\right) d s\right]
\end{aligned}
$$

where $K_{2}(q)$ is the constant appearing in Lemma 4.2, and $\alpha(A, q)$ is the number defined by (4.30). 
Proof. By the monotone convergence theorem for integrals, it suffices to prove that

$$
\begin{gathered}
\int_{0}^{\infty} \lambda \chi_{[0, t)}(s) \sum_{i=-N}^{N-1} \lambda_{i} \chi_{\left[t_{i}, t_{i+1}\right)}(s) d s \leq 2 K_{2}(q)\left[\int_{0}^{\infty} B_{A, q}\left(\lambda s^{-1 / q} \chi_{[0, t)}(s)\right) d s\right. \\
\left.+\int_{0}^{\infty} \widetilde{A}\left(s^{-1 / q^{\prime}} \int_{0}^{s} \sum_{i=-N}^{N-1} \lambda_{i} \chi_{\left[t_{i}, t_{i+1}\right)}(r) d r\right) d s\right]
\end{gathered}
$$

for $\lambda, \lambda_{i}, t, t_{i}$ as in the statement and for every $N \in \mathbb{N}$.

We claim that inequality (4.41) is a consequence of

$$
\begin{gathered}
\int_{0}^{\infty} \lambda \chi_{[0, t)}(s) \sum_{i=-N}^{N-1} \lambda_{i} \chi_{\left[t_{i}, t_{i+1}\right)}(s) d s \leq 2 K_{2}(q)\left\|\lambda s^{-1 / q} \chi_{[0, t)}(s)\right\|_{L^{B} A, q(0, \infty)} \\
\times\left\|s^{-1 / q^{\prime}} \int_{0}^{s} \sum_{i=-N}^{N-1} \lambda_{i} \chi_{\left[t_{i}, t_{i+1}\right)}(r) d r\right\|_{L^{\tilde{A}}(0, \infty)}
\end{gathered}
$$

for $t \geq \alpha(A, q) / 2$. To see this, set

$$
M=\int_{0}^{\infty} B_{A, q}\left(\lambda s^{-1 / q} \chi_{[0, t)}(s)\right) d s+\int_{0}^{\infty} \widetilde{A}\left(s^{-1 / q^{\prime}} \int_{0}^{s} \sum_{i=-N}^{N-1} \lambda_{i} \chi_{\left[t_{i}, t_{i+1}\right)}(r) d r\right) d s,
$$

apply (4.42) with $A(s)$ replaced by $A^{M}(s)=A(s) / M$, and observe that, after this replacement, $B_{A, q}(s)$ is changed into $B_{A^{M}, q}(s)=B_{A, q}(s) / M, \widetilde{A}(s)$ into $\widetilde{A^{M}}(s)=\widetilde{A}(M s) / M$ and $\alpha(A, q)$ into $\alpha\left(A^{M}, q\right)=M \alpha(A, q)$. Consequently,

$$
\left\|\lambda s^{-1 / q} \chi_{[0, t)}(s)\right\|_{L^{B} A^{M, q}(0, \infty)} \leq 1
$$

and

$$
\left\|s^{-1 / q^{\prime}} \int_{0}^{s} \sum_{i=-N}^{N-1} \lambda_{i} \chi_{\left[t_{i}, t_{i+1}\right)}(r) d r\right\|_{L^{\widetilde{A^{M}}}(0, \infty)} \leq M,
$$

whence (4.41) follows.

Let us establish (4.42). We may clearly suppose that $\lambda=1$. We have

$$
\begin{aligned}
& \int_{0}^{\infty} \chi_{[0, t)}(s) \sum_{i=-N}^{N-1} \lambda_{i} \chi_{\left[t_{i}, t_{i+1}\right)}(s) d s= \\
& \quad= \begin{cases}0 & \text { if } t \leq t_{-N} \\
\sum_{i=-N}^{j-1} \lambda_{i}\left(t_{i+1}-t_{i}\right)+\lambda_{j}\left(t-t_{j}\right) & \text { if } t \in\left(t_{j}, t_{j+1}\right] \text { for some } \\
\sum_{i=-N}^{N-1} \lambda_{i}\left(t_{i+1}-t_{i}\right) & j \in\{-N, \ldots, N-1\}\end{cases}
\end{aligned}
$$


When $t \leq t_{N}$ there is nothing to prove. Assume that $t \in\left(t_{j}, t_{j+1}\right]$ for some $j \in\{-N, \ldots, N-1\}$. Then

$$
\begin{aligned}
& \left\|s^{-1 / q^{\prime}} \int_{0}^{s} \sum_{i=-N}^{N-1} \lambda_{i} \chi_{\left[t_{i}, t_{i+1}\right)}(r) d r\right\|_{L^{\tilde{A}}(0, \infty)} \\
& =\left\|s^{-1 / q^{\prime}} \sum_{i=-N}^{N-1}\left(\lambda_{i}\left(s-t_{i}\right) \chi_{\left[t_{i}, t_{i+1}\right)}(s)+\lambda_{i}\left(t_{i+1}-t_{i}\right) \chi_{\left[t_{i+1}, \infty\right)}(s)\right)\right\|_{L^{\tilde{A}}(0, \infty)} \\
& \geq \| s^{-1 / q^{\prime}}\left(\sum_{i=-N}^{j-1} \lambda_{i}\left(t_{i+1}-t_{i}\right) \chi_{\left[t_{i+1}, \infty\right)}(s)+\lambda_{j}\left(s-t_{j}\right) \chi_{\left[t_{j}, t\right)}(s)\right. \\
& \left.\quad+\lambda_{j}\left(s-t_{j}\right) \chi_{\left[t, t_{j+1}\right)}(s)+\lambda_{j}\left(t_{j+1}-t_{j}\right) \chi_{\left[t_{j+1}, \infty\right)}(s)\right) \|_{L^{\tilde{A}}(0, \infty)} \\
& \geq s^{-1 / q^{\prime}}\left(\sum_{i=-N}^{j-1} \lambda_{i}\left(t_{i+1}-t_{i}\right) \chi_{[t, \infty)}(s)+\lambda_{j}\left(t-t_{j}\right) \chi_{\left[t, t_{j+1}\right)}(s)\right. \\
& \left.\quad+\lambda_{j}\left(t-t_{j}\right) \chi_{\left[t_{j+1}, \infty\right)}(s)\right) \|_{L^{\tilde{A}}(0, \infty)} \\
& =\left(\sum_{i=-N}^{j-1} \lambda_{i}\left(t_{i+1}-t_{i}\right)+\lambda_{j}\left(t-t_{j}\right)\right)\left\|_{s^{-1 / q^{\prime}} \chi_{[t, \infty)}(s)}\right\|_{L^{\tilde{A}(0, \infty)}}
\end{aligned}
$$

If $t>t_{N}$, then we have

$$
\begin{aligned}
& \left\|s^{-1 / q^{\prime}} \int_{0}^{s} \sum_{i=-N}^{N-1} \lambda_{i} \chi_{\left[t_{i}, t_{i+1}\right)}(r) d r\right\|_{L^{\tilde{A}}(0, \infty)} \\
& =\left\|s^{-1 / q^{\prime}} \sum_{i=-N}^{N-1}\left(\lambda_{i}\left(s-t_{i}\right) \chi_{\left[t_{i}, t_{i+1}\right)}(s)+\lambda_{i}\left(t_{i+1}-t_{i}\right) \chi_{\left[t_{i+1}, \infty\right)}(s)\right)\right\|_{L^{\tilde{A}}(0, \infty)} \\
& \geq\left\|s^{-1 / q^{\prime}} \sum_{i=-N}^{N-1} \lambda_{i}\left(t_{i+1}-t_{i}\right) \chi_{\left[t_{i+1}, \infty\right)}(s)\right\|_{L^{\tilde{A}}(0, \infty)} \\
& \geq\left\|s^{-1 / q^{\prime}} \sum_{i=-N}^{N-1} \lambda_{i}\left(t_{i+1}-t_{i}\right) \chi_{[t, \infty)}(s)\right\|_{L^{\tilde{A}}(0, \infty)} \\
& =\sum_{i=-N}^{N-1} \lambda_{i}\left(t_{i+1}-t_{i}\right)\left\|s^{-1 / q^{\prime}} \chi_{[t, \infty)}(s)\right\|_{L^{\tilde{A}}(0, \infty)}
\end{aligned}
$$

Owing to Lemma 4.3, inequality (4.42) follows from (4.43) and (4.44) if $t \in\left(t_{j}, t_{j+1}\right]$ for some $j \in\{-N, \ldots, N-1\}$, and from (4.43) and (4.45) if $t>t_{N}$. 
Proof of Theorem 4.1. The statement will be proved with $\alpha(A, q)$ defined by (4.30). For simplicity of notation, throughout the proof we denote $B_{A, q}$ by $B$. Owing to the Hardy-Littlewood inequality (2.5), we may assume that $f=f^{*}$ and $g=g^{*}$. We claim that inequality (4.2) will follow if we show that

$$
\begin{aligned}
\int_{0}^{\infty} f^{*}(s) g^{*}(s) d s \leq K_{1}(q) & \left(\int_{0}^{\infty} B\left(s^{-1 / q} f^{*}(s)\right) d s\right. \\
& \left.+\int_{0}^{\infty} \widetilde{A}\left(s^{-1 / q^{\prime}} \int_{0}^{s} g^{*}(r) d r\right) d s\right)
\end{aligned}
$$

for any $f$ and $g$ as in the statement and satisfying, in addition,

$$
\int_{0}^{\infty} B\left(s^{-1 / q} f^{*}(s)\right) d s \leq 1 \text { and } \int_{0}^{\infty} \widetilde{A}\left(s^{-1 / q^{\prime}} \int_{0}^{s} g^{*}(r) d r\right) d s \leq 1 .
$$

To verify our claim, notice that if $f$ and $g$ are any functions making the right-hand side of (4.2) finite, then the new functions $\bar{f}$ and $\bar{g}$, defined for $\epsilon>0$ as

$\bar{f}(s)=\frac{f^{*}(s)}{\left\|(\cdot)^{-1 / q} f^{*}(\cdot)\right\|_{L^{B}(0, \infty)}+\epsilon}$ and $\bar{g}(s)=\frac{g^{*}(s)}{\left\|(\cdot)^{-1 / q^{\prime}} \int_{0}^{\cdot} g^{*}(r) d r\right\|_{L^{\tilde{A}}(0, \infty)}+\epsilon}$,

satisfy (4.47); notice also that $\bar{f}$ fulfills (4.4) if $f$ does. Moreover, an application of (4.46) with $f^{*}$ and $g^{*}$ replaced by $\bar{f}$ and $\bar{g}$ yields

$$
\begin{aligned}
& \int_{0}^{\infty} \frac{f^{*}(s) g^{*}(s)}{\left(\left\|(\cdot)^{-1 / q} f^{*}(\cdot)\right\|_{L^{B}(0, \infty)}+\epsilon\right)\left(\left\|(\cdot)^{-1 / q^{\prime}} \int_{0}^{\cdot} g^{*}(r) d r\right\|_{L^{\tilde{A}}(0, \infty)}+\epsilon\right)} d s \leq \\
& \leq \frac{K_{1}(q)}{2}\left(\int_{0}^{\infty} B\left(\frac{s^{-1 / q} f^{*}(s)}{\left\|(\cdot)^{-1 / q} f^{*}(\cdot)\right\|_{L^{B}(0, \infty)}+\epsilon}\right) d s\right. \\
&\left.\quad+\int_{0}^{\infty} \widetilde{A}\left(\frac{s^{-1 / q^{\prime}} \int_{0}^{s} g^{*}(r) d r}{\left\|(\cdot)^{-1 / q^{\prime}} \int_{0}^{\cdot} g^{*}(r) d r\right\|_{L^{\tilde{A}}(0, \infty)}+\epsilon}\right) d s\right) \\
&(4.48) \leq K_{1}(q) .
\end{aligned}
$$

Inequality (4.48) implies (4.2), thanks to the arbitrariness of $\epsilon$.

We now prove (4.46). For every $k \in \mathbb{Z}$ such that

$$
2^{k+1} \leq \operatorname{ess} \sup |f|
$$

define the non-increasing function $f_{k}:[0, \infty) \rightarrow[0, \infty)$ as

$$
f_{k}(s)= \begin{cases}0 & \text { if } f^{*}(s)<2^{k} \\ f^{*}(s)-2^{k} & \text { if } 2^{k} \leq f^{*}(s)<2^{k+1} \\ 2^{k} & \text { if } f^{*}(s) \geq 2^{k+1}\end{cases}
$$


Furthermore, set

$$
F_{k}=\left\{s \geq 0: 2^{k+1} \leq f^{*}<2^{k+2}\right\}
$$

and, for $k, m \in \mathbb{Z}$,

$$
G_{k, m}=\left\{s \in F_{k}: 2^{m} \leq g^{*}<2^{m+1}\right\} .
$$

Note that each of the sets $F_{k}$ and $G_{k, m}$ is either empty or an interval, and that $F_{k}=\cup_{m \in \mathbb{Z}} G_{k, m}$. Furthermore, $F_{k} \subseteq\left\{f^{*} \geq 2^{k+1}\right\}=\left\{f_{k} \geq 2^{k}\right\}$, an interval whose endpoints are 0 and $\left|\left\{f_{k} \geq 2^{k}\right\}\right|$. One has,

$$
\begin{aligned}
\int_{0}^{\infty} f^{*}(s) g^{*}(s) d s=\int_{0}^{\infty}\left(\sum_{k} \chi_{F_{k}}(s) f^{*}(s)\right) g^{*}(s) d s \\
=\sum_{k} \int_{0}^{\infty} \chi_{F_{k}}(s) f^{*}(s) g^{*}(s) d s \leq \sum_{k} \int_{0}^{\infty} 2^{k+2} \chi_{F_{k}}(s) g^{*}(s) d s \\
=\sum_{k} \int_{0}^{\infty} 2^{k+2} \chi_{\left\{f_{k} \geq 2^{k}\right\}}(s) \chi_{F_{k}}(s) g^{*}(s) d s \\
=\sum_{k} \int_{0}^{\infty} 2^{k+2} \chi_{\left\{f_{k} \geq 2^{k}\right\}}(s) \sum_{m} \chi_{G_{k, m}}(s) g^{*}(s) d s \\
\quad \leq \sum_{k} 8 \int_{0}^{\infty} 2^{k} \chi_{\left\{f_{k} \geq 2^{k}\right\}}(s) \sum_{m} 2^{m} \chi_{G_{k, m}}(s) d s .
\end{aligned}
$$

By Lemma 4.4, we have that, for every $k$ satisfying (4.49),

$$
\begin{gathered}
\int_{0}^{\infty} 2^{k} \chi_{\left\{f_{k} \geq 2^{k}\right\}}(s) \sum_{m} 2^{m} \chi_{G_{k, m}}(s) d s \leq 2 K_{2}(q)\left[\int_{0}^{\infty} B\left(2^{k} s^{-1 / q} \chi_{\left\{f_{k} \geq 2^{k}\right\}}(s)\right) d s\right. \\
\left.+\int_{0}^{\infty} \widetilde{A}\left(s^{-1 / q^{\prime}} \int_{0}^{s} \sum_{m} 2^{m} \chi_{G_{k, m}}(r) d r\right) d s\right] .
\end{gathered}
$$

Observe that $t=\left|\left\{f_{k} \geq 2^{k}\right\}\right|$ in this application of Lemma 4.4. Thus, we need to know that

$$
\begin{aligned}
& \left|\left\{f_{k} \geq 2^{k}\right\}\right| \geq \frac{\alpha(A, q)}{2}\left[\int_{0}^{\infty} B\left(2^{k} s^{-1 / q} \chi_{\left\{f_{k} \geq 2^{k}\right\}}(s)\right) d s\right. \\
& \left.+\int_{0}^{\infty} \widetilde{A}\left(s^{-1 / q^{\prime}} \int_{0}^{s} \sum_{m} 2^{m} \chi_{G_{k, m}}(r) d r\right) d s\right] .
\end{aligned}
$$

Since (4.49) is in force, then

$$
\left|\left\{f_{k} \geq 2^{k}\right\}\right|=\left|\left\{f^{*} \geq 2^{k+1}\right\}\right|=\left|\left\{f \geq 2^{k+1}\right\}\right| \geq \alpha(A, q),
$$

by assumption (4.4). 
Moreover, inasmuch as

$$
2^{k} \chi_{\left\{f_{k} \geq 2^{k}\right\}}(s) \leq f_{k}(s) \leq f^{*}(s) \text { for } s>0,
$$

$$
\sum_{m} 2^{m} \chi_{G_{k, m}}(s) \leq \sum_{m} g^{*}(s) \chi_{G_{k, m}}(s)=g^{*}(s) \chi_{F_{k}}(s) \leq g^{*}(s) \text { for } s>0,
$$

and we are assuming (4.47), then

$$
\begin{aligned}
& \int_{0}^{\infty} B\left(2^{k} s^{-1 / q} \chi_{\left\{f_{k} \geq 2^{k}\right\}}(s)\right) d s+\int_{0}^{\infty} \widetilde{A}\left(s^{-1 / q^{\prime}} \int_{0}^{s} \sum_{m} 2^{m} \chi_{G_{k, m}}(r) d r\right) d s \\
& \quad \leq \int_{0}^{\infty} B\left(s^{-1 / q} f^{*}(s)\right) d s+\int_{0}^{\infty} \widetilde{A}\left(s^{-1 / q^{\prime}} \int_{0}^{s} g^{*}(r) d r\right) d s \leq 2
\end{aligned}
$$

Inequality (4.53) is a consequence of (4.54) and (4.57).

Now, by (4.55)-(4.56),

$$
\begin{gathered}
\int_{0}^{\infty} B\left(2^{k} s^{-1 / q} \chi_{\left\{f_{k} \geq 2^{k}\right\}}(s)\right) d s+\int_{0}^{\infty} \widetilde{A}\left(s^{-1 / q^{\prime}} \int_{0}^{s} \sum_{m} 2^{m} \chi_{G_{k, m}}(r) d r\right) d s \\
\quad \leq \int_{0}^{\infty} B\left(s^{-1 / q} f_{k}(s)\right) d s+\int_{0}^{\infty} \widetilde{A}\left(s^{-1 / q^{\prime}} \int_{0}^{s} g^{*}(r) \chi_{F_{k}}(r) d r\right) d s .
\end{gathered}
$$

Combining (4.51), (4.52) and (4.58) yields, via the monotone convergence theorem for integrals,

$$
\begin{aligned}
\int_{0}^{\infty} f^{*}(s) g^{*}(s) d s \leq & 16 K_{2}(q)\left[\int_{0}^{\infty} \sum_{k} B\left(s^{-1 / q} f_{k}(s)\right) d s\right. \\
& \left.+\int_{0}^{\infty} \sum_{k} \widetilde{A}\left(s^{-1 / q^{\prime}} \int_{0}^{s} g^{*}(r) \chi_{F_{k}}(r) d r\right) d s\right]
\end{aligned}
$$

By property (2.13) and by monotone convergence again, the right-hand side of (4.59) does not exceed

$$
16 K_{2}(q)\left[\int_{0}^{\infty} B\left(s^{-1 / q} \sum_{k} f_{k}(s)\right) d s+\int_{0}^{\infty} \widetilde{A}\left(s^{-1 / q^{\prime}} \int_{0}^{s} g^{*}(r) \sum_{k} \chi_{F_{k}}(r) d r\right) d s\right] .
$$

Since

$$
\sum_{k} f_{k}(s)=f^{*}(s) \text { and } \sum_{k} \chi_{F_{k}}(s)=1 \quad \text { for } \quad s \geq 0
$$

inequality (4.46) follows with $K_{1}(q)=16 K_{2}(q)$. 


\section{Comparison between $A$ and $B_{A, q}$}

The present section is devoted to the study of relations between a given Young function $A$, satisfying (1.17) for some $q \in(1, \infty)$, and the Young function $B_{A, q}$ associated with $A$ and $q$ as in Definition 1.2. Our first observation, contained in Proposition 5.1, is that $A$ always dominates $B_{A, q}$. In subsequent Proposition 5.2, the couples $(A, q)$ for which $A$ and $B_{A, q}$ are equivalent are described in terms of the Matuzewska-Orlicz indices of $A$. As a consequence, the cases of equality of the spaces $L\left(q, B_{A, q}\right)(G)$ and $L(q, A)(G)$, and of related spaces, are characterized.

Proposition 5.1 Let $q \in(1, \infty)$ and let $A$ be a Young function satisfying (1.17). Then $A$ dominates $B_{A, q}$.

Proof. We have to prove that there exists a positive constant $c$ such that $B_{A, q}(s) \leq A(c s)$ for $s \geq 0$. Owing to (2.11) and to corresponding estimates for $B_{A, q}$, this inequality is equivalent to $b(s) \leq k a(k s)$ for $s \geq 0$ and for some constant $k$, where $b$ is given as in Definition 2.11; the latter inequality is in turn equivalent to

$$
k b^{-1}(r) \geq a^{-1}(r / k) \quad \text { for } r \geq 0 .
$$

Inequality (5.1) reads

$$
\left(\int_{a^{-1}(s)}^{\infty}\left(\int_{0}^{t}\left(\frac{1}{a(r)}\right)^{\frac{1}{q-1}} d r\right)^{-q} \frac{d t}{a(t)^{q^{\prime}}}\right)^{\frac{1}{1-q}} \geq \frac{1}{k} a^{-1}\left(\frac{s}{k}\right) \text { for } s \geq 0 .
$$

Since

$$
\left(\frac{1}{a(r)}\right)^{1 /(q-1)}
$$

is non-increasing in $(0, \infty)$, then

$$
\frac{1}{t} \int_{0}^{t}\left(\frac{1}{a(r)}\right)^{1(q-1)} d r \geq\left(\frac{1}{a(t)}\right)^{1 /(q-1)}
$$

for $t>0$. Consequently,

$$
\int_{a^{-1}(s)}^{\infty}\left(\int_{0}^{t}\left(\frac{1}{a(r)}\right)^{\frac{1}{q-1}} d r\right)^{-q} \frac{d t}{a(t)^{q^{\prime}}} \leq \int_{a^{-1}(s)}^{\infty} t^{-q} d t=\frac{\left(a^{-1}(s)\right)^{1-q}}{q-1}
$$

Hence, (5.2) follows with $k=\max \left\{1,(q-1)^{-1 /(q-1)}\right\}$. 
Proposition 5.2 Let $q \in(1, \infty)$ and let $A$ be a Young function. Let $n \geq 1$. I. Assume that (1.17) is fulfilled. Then the following assertions are equivalent:

(i) $B_{A, q}$ is equivalent to $A$;

(ii) $A$ is finite-valued and $I(A)<q$;

(iii) If $G$ is any measurable subset of $\mathbb{R}^{n}$ having infinite measure, then $L\left(q, B_{A, q}\right)(G)=L(q, A)(G)$ (up to equivalent norms).

II. Assume that (1.17) is fulfilled. Let $A^{0}$ be any Young function which agrees with $A$ near 0 (in particular, the choice $A^{0}=A$ is admissible). Then the following assertions are equivalent:

(i) $B_{A^{0}, q}$ is equivalent to $A$ near 0 ;

(ii) $I_{0}(A)<q$;

(iii) If $G$ is any measurable subset of $\mathbb{R}^{n}$ having infinite measure and $\widehat{A}$ is any Young function which agrees with $A$ near 0 and satisfies

$$
\int^{\infty} \frac{\widehat{A}(r)}{r^{1+q}} d r<\infty
$$

then $L\left(q, B_{A^{0}, q}\right)(G) \cap L^{\infty}(G)=L(q, \widehat{A})(G) \cap L^{\infty}(G)$ (up to equivalent norms).

III. Let $A^{\infty}$ be any Young function which agrees with $A$ near infinity and satisfies

$$
\int_{0}\left(\frac{r}{A^{\infty}(r)}\right)^{1 /(q-1)} d r<\infty
$$

(in particular, the choice $A^{\infty}=A$ is admissible if $A$ fulfills (1.17)). Then the following assertions are equivalent:

(i) $B_{A^{\infty}, q}$ is equivalent to $A$ near infinity;

(ii) $A$ is finite-valued and $I_{\infty}(A)<q$;

(iii) If $G$ is any measurable subset of $\mathbb{R}^{n}$ having finite measure, then

$$
L\left(q, B_{A^{\infty}, q}\right)(G)=L(q, A)(G)
$$

(up to equivalent norms). 
Proof. Part I. (ii) implies (i). Since $A$ always dominates $B_{A, q}$, we have only to show that $B_{A, q}$ dominates $A$. It is easily seen, thanks to inequalities (2.11), that $I(a)=I(A)-1$, whence $I(a)<q-1$. A well-known property of Matuzewska-Orlicz indices (see e.g. [6]) tells us that, for every $\gamma>I(a)$, a constant $C$ exists such that

$$
a(s) \leq C a(r)\left(\frac{s}{r}\right)^{\gamma} \text { if } 0 \leq r \leq s .
$$

Now choose $\gamma \in(I(a), q-1)$. Then

$$
\begin{aligned}
\frac{1}{t} \int_{0}^{t}\left(\frac{1}{a(r)}\right)^{\frac{1}{q-1}} d r & \leq \frac{1}{t} C^{\frac{1}{q-1}}\left(\frac{t^{\gamma}}{a(t)}\right)^{\frac{1}{q-1}} \int_{0}^{t} r^{\frac{\gamma}{1-q}} d r \\
& =C^{\frac{1}{q-1}}\left(\frac{q-1}{q-1-\gamma}\right)\left(\frac{1}{a(t)}\right)^{\frac{1}{q-1}} \text { for } t>0 .
\end{aligned}
$$

Thus, a positive constant $C_{1}$ exists such that

$$
\int_{a^{-1}(s)}^{\infty}\left(\int_{0}^{t}\left(\frac{1}{a(r)}\right)^{\frac{1}{q-1}} d r\right)^{-q} \frac{d t}{a(t)^{q^{\prime}}} \geq C_{1} \int_{a^{-1}(s)}^{\infty} t^{-q} d t=C_{1} \frac{\left(a^{-1}(s)\right)^{1-q}}{q-1}
$$

for $t>0$, whence $b^{-1}(s) \leq\left((q-1) / C_{1}\right)^{1 /(q-1)} a^{-1}(s)$ for $s \geq 0$. This inequality tells us that $B_{A, q}$ dominates $A$.

(iii) implies (ii). Assumption (iii) clearly ensures that $L(q, A)(0, \infty)$ is an r.i. space, and, by $(2.10)$, also that $L(q, A)(0, \infty)=L\left(q, B_{A, q}\right)(0, \infty)$ with equivalent norms. Therefore, a constant $C_{2}$ exists such that

$$
\|f\|_{L(q, A)(0, \infty)} \leq C_{2}\|f\|_{L\left(q, B_{A, q}\right)(0, \infty)}
$$

for every $f \in L\left(q, B_{A, q}\right)(0, \infty)$. Combining this inequality with inequality (3.1) (with $n$ replaced by $q$ ) tells us that there exists a constant $C_{3}$ such that

$$
\left\|s^{-1 / q} \int_{s}^{\infty} r^{-1 / q^{\prime}} f(r) d r\right\|_{L^{A}(0, \infty)} \leq C_{3}\|f(s)\|_{L^{A}(0, \infty)}
$$

for every $f \in L^{A}(0, \infty)$. Hence, owing to [13, Lemma 5], a constant $k$ exists such that

$$
\left(\int_{k s}^{\infty}\left(\frac{r}{\widetilde{A}(r)}\right)^{q-1} d r\right)^{1 / q}\left(\int_{0}^{s} \frac{\widetilde{A}(r)}{r^{1+q^{\prime}}} d r\right)^{1 / q^{\prime}} \leq k s \quad \text { for } s \geq 0 .
$$

Lemma 4 of the same paper then entails that $I\left(\widetilde{A}^{-1}\right)<1 / q^{\prime}$, whence $i\left(A^{-1}\right)>1 / q$, since $i\left(A^{-1}\right)+I\left(\widetilde{A}^{-1}\right)=1$ (see [7]). Such an estimate 
for $i\left(A^{-1}\right)$ ensures that $A$ is finite-valued. In fact, if $A(s)$ were equal to $\infty$ for large $s$, then $A^{-1}(r)$ would be constant for large $r$, whence $i\left(A^{-1}\right)=0$. Moreover, since $A(s)$ is also strictly positive for $s>0$ by assumption (1.17), then $A$ is strictly increasing. Hence, the condition $i\left(A^{-1}\right)>1 / q$ is equivalent to $I(A)<q$ (see [7] again). Thus, (ii) follows.

(i) implies (iii). This implication is a straightforward consequence of the definition of the norms in $L(q, A)(G)$ and $L\left(q, B_{A, q}\right)(G)$, and of the fact that Luxemburg norms associated with equivalent Young functions are equivalent.

Part II. (ii) implies (i). Condition (ii) is equivalent to $I_{0}(a)<q-1$. Moreover, given any $\gamma>I_{0}(a)$, constants $C$ and $s_{0}$ exist such that inequality (5.3) holds if $0<r \leq s \leq s_{0}$. One can then proceed analogously as in Part I and show that $B_{A, q}$ dominates $A$ near 0 , and hence that $B_{A, q}$ is equivalent to $A$ near 0 . It is not difficult to verify that $B_{A^{0}, q}$ is equivalent to $B_{A, q}$ near 0. Consequently, (i) follows.

(iii) implies (ii). Since we are assuming that $\int^{\infty} \frac{\widehat{A}(r)}{r^{1+q}} d r<\infty$, then Proposition 2.1 ensures that $L(q, \widehat{A})(0, \infty) \cap L^{\infty}(0, \infty)$ is an r.i. space, which agrees with $L\left(q, B_{A^{0}, q}\right)(0, \infty) \cap L^{\infty}(0, \infty)$, by (iii). In particular, by $(2.10)$, a positive constant $C_{1}$ exists such that

$$
\|f\|_{L(q, \widehat{A})(0, \infty) \cap L^{\infty}(0, \infty)} \leq C_{1}\|f\|_{L\left(q, B_{A^{0}, q}\right)(0, \infty) \cap L^{\infty}(0, \infty)}
$$

for every $f \in L\left(q, B_{A^{0}, q}\right)(0, \infty) \cap L^{\infty}(0, \infty)$. Now, let $\check{A}$ be any Young function which agrees with $A$ (and hence with $A^{0}$ ) near 0 and satisfies

$$
\int^{\infty}\left(\frac{r}{\check{A}(r)}\right)^{1 /(q-1)} d r<\infty .
$$

Then $B_{\breve{A}, q}$ is equivalent to $B_{A^{0}, q}$ near 0, and the functions $E_{B_{\check{A}, q}}$ and $E_{B_{A^{0}, q}}$, defined as in (2.19), are globally equivalent. Hence, by Proposition 2.1, Part II,

$$
L\left(q, B_{\check{A}, q}\right)(0, \infty) \cap L^{\infty}(0, \infty)=L\left(q, B_{A^{0}, q}\right)(0, \infty) \cap L^{\infty}(0, \infty) ;
$$

therefore, a constant $C_{2}$ exists such that

$$
\|f\|_{L\left(q, B_{A^{0}, q}\right)(0, \infty) \cap L^{\infty}(0, \infty)} \leq C_{2}\|f\|_{L\left(q, B_{\check{A}, q}\right)(0, \infty) \cap L^{\infty}(0, \infty)}
$$

for every $f \in L\left(q, B_{\check{A}, q}\right)(0, \infty) \cap L^{\infty}(0, \infty)$. By inequalities (3.1)-(3.2) (with $A$ replaced by $\check{A}$ and $n$ replaced by $q$ ), a constant $C_{3}$ exists such that

$$
\left\|\int_{s}^{\infty} r^{-1 / q^{\prime}} f(r) d r\right\|_{L\left(q, B_{\check{A}, q}\right)(0, \infty)}+\left\|\int_{s}^{\infty} r^{-1 / q^{\prime}} f(r) d r\right\|_{L^{\infty}(0, \infty)} \leq C_{3}\|f\|_{L^{\check{A}}(0, \infty)}
$$

for every $f \in L^{\check{A}}(0, \infty)$. 
From (5.6)-(5.8), we deduce that

$$
\begin{aligned}
\| s^{-1 / q} \int_{s}^{\infty} r^{-1 / q^{\prime}} & f(r) d r\left\|_{L^{\widehat{A}}(0, \infty)} \leq\right\| \int_{s}^{\infty} r^{-1 / q^{\prime}}|f(r)| d r \|_{L(q, \widehat{A})(0, \infty)} \\
& \leq\left\|\int_{s}^{\infty} r^{-1 / q^{\prime}}|f(r)| d r\right\| \|_{L(q, \widehat{A})(0, \infty) \cap L^{\infty}(0, \infty)} \\
& \leq C_{1} C_{2}\left\|\int_{s}^{\infty} r^{-1 / q^{\prime}}|f(r)| d r\right\|_{\left.L\left(q, B_{\check{A}, q}\right)(0, \infty)\right) \cap L^{\infty}(0, \infty)} \\
& \leq C_{1} C_{2} C_{3}\|f\|_{L^{\check{A}}(0, \infty)}
\end{aligned}
$$

for every $f \in L^{\check{A}}(0, \infty)$. Owing to [13, Lemma 5], one infers from (5.9) that a constant $k$ exists such that

$$
\left(\int_{k s}^{\infty}\left(\frac{r}{\widetilde{\widehat{A}}(r)}\right)^{q-1} d r\right)^{1 / q}\left(\int_{0}^{s} \frac{\widetilde{A}(r)}{r^{1+q^{\prime}}} d r\right)^{1 / q^{\prime}} \leq k \quad \text { for } s \geq 0 .
$$

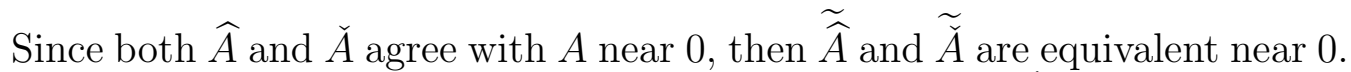
This ensures that (5.10) also holds, with $\check{A}$ replaced by $\widehat{A}$ (possibly with a different constant $k$ ) for small $s$. Therefore, via analogous arguments as in the proof of [13, Lemma 4], one can deduce that

$$
I_{0}\left(\widetilde{\widehat{A}}^{-1}\right)<1 / q^{\prime}
$$

and hence that $I_{0}(A)=I_{0}(\widehat{A})<q$.

(i) implies (iii). Since $B_{A^{0}, q}$ is equivalent to $A$ near 0 , and $A$ agrees with $\widehat{A}$ near 0 , then $B_{A^{0}, q}$ is equivalent to $\widehat{A}$ near 0 . Thus, the functions $E_{B_{A^{0}, q}}$ and $E_{\widehat{A}}$, defined as in (2.19), are globally equivalent. Consequently, $L\left(q ; B_{A^{0}, q}, \infty\right)(G)=L(q ; \widehat{A}, \infty)(G)$. By Proposition 2.1, Part II, assertion (iii) follows.

Part III. (ii) implies (i). Analogously as in Parts I and II, we have that $I^{\infty}(a)<q-1$ and that, given any $\gamma>I^{\infty}(a)$, constants $C$ and $s_{\infty}$ exist such that inequality (5.3) holds if $s_{\infty} \leq r \leq s$. Hence, it is not difficult to show that there exists a constant $C_{1}$ such that

$$
\frac{1}{t} \int_{0}^{t}\left(\frac{1}{a^{\infty}(r)}\right)^{\frac{1}{q-1}} d r \leq C_{1}\left(\frac{1}{a^{\infty}(t)}\right)^{\frac{1}{q-1}}
$$

for large $t$, where $a^{\infty}$ is the non decreasing left-continuous function such that $A^{\infty}(s)=\int_{0}^{s} a^{\infty}(r) d r$. Since $a^{\infty}(s)=a(s)$ for large $s$, one can infer 
from (5.11) that $b^{-1}(r) \leq C_{2} a^{-1}(r)$ for some constant $C_{2}$ and for sufficiently large $r$. Then $B_{A, q}$ dominates $A$ near infinity and, by Proposition 5.1, it is in fact equivalent to $A$ near infinity.

(iii) implies (ii). From inequality (3.1), applied with $n$ and $A$ replaced by $q$ and $A^{\infty}$, respectively, and from the fact that $A^{\infty}$ agrees with $A$ near infinity we get that a constant $C_{2}$, depending only on $A, n$ and $|G|$ exists such that

$$
\left\|s^{-1 / q} \int_{s}^{\infty} r^{-1 / q^{\prime}} f(r) d r\right\|_{L^{A^{\infty}(0,|G|)}} \leq C_{2}\|f\|_{L^{A^{\infty}}(0,|G|)}
$$

for every $f \in L^{A^{\infty}}(0,|G|)$. [13, Theorem 5] then tells us that there exists a constant $k$ such that

$$
\left(\int_{k s}^{\infty}\left(\frac{r}{\widetilde{A^{\infty}}(r)}\right)^{q-1} d r\right)^{1 / q}\left(\int_{0}^{s} \frac{\widetilde{A^{\infty}}(r)}{r^{1+q^{\prime}}} d r\right)^{1 / q^{\prime}} \leq k
$$

for large $s$. Since $A$ equals $A^{\infty}$ near infinity, then inequality (5.12) also holds with $A^{\infty}$ replaced by $A$ (possibly with a different constant $k$ ) for large $s$. Hence, by [13, Lemma 4], $I_{\infty}(\widetilde{A})<1 / q^{\prime}$, whence we conclude as above that $I_{\infty}(A)<q$.

(i) implies (iii). Since $B_{A^{\infty}, q}$ is equivalent to $A$ near infinity and, by Proposition 2.2 applied to $A^{\infty}$,

$$
\int^{\infty} \frac{B_{A^{\infty}, q}(t)}{t^{1+q}} d t<\infty, \quad \text { then } \int^{\infty} \frac{A(t)}{t^{1+q}} d t<\infty
$$

Hence, $L(q, A)(G)$ is an r.i. space. The equation $L\left(q, B_{A^{\infty}, q}\right)(G)=L(q, A)(G)$ follows from the equivalence of Luxemburg norms over sets of finite measure associated with Young functions equivalent near infinity.

\section{References}

[1] Adams, R. A.: On the Orlicz-Sobolev imbedding theorem. J. Functional Analysis 24 (1977), 241-257.

[2] Alvino, A., Ferone, V. and Trombetti, G.: Moser-type inequalities in Lorentz spaces. Potential Anal. 5 (1996), no. 3, 273-299.

[3] Bennett, C. and Rudnick, K.: On Lorentz-Zygmund spaces. Dissertationes Math. 175 (1980), 1-72.

[4] Bennett, C. and Sharpley, R.: Interpolation of operators. Pure and Applied Mathematics 129. Academic Press, Boston, 1988. 
[5] Besov, O. V., Il'in, V.P. And Nikolskit, S. M.: Integral representations of functions and embedding theorems. Nauka, Moscow, 1975; English translation: Wiley, New York-Toronto, 1979.

[6] Bingham, N. H., Goldie, C. M. and Teugels, J. L.: Regular variation. Encyclopedia of Mathematics and its Applications 27. Cambridge University Press, Cambridge, 1987.

[7] Boyd, D. W.: Indices for the Orlicz spaces. Pacific J. Math. 38 (1971), 315-323.

[8] Brezis, H. And Wainger, S.: A note on limiting cases of Sobolev embeddings and convolution inequalities. Comm. Partial Differential Equations 5 (1980), no. 7, 773-789.

[9] Brothers, J. E. And Ziemer, W. P.: Minimal rearrangements of Sobolev functions. J. Reine Angew. Math 384 (1988), 153-179.

[10] Cianchi, A.: A sharp embedding theorem for Orlicz-Sobolev spaces. Indiana Univ. Math. J. 45 (1996), no. 1, 39-65.

[11] Cianchi, A.: Boundedness of solutions to variational problems under general growth conditions. Comm. Partial Differential Equations 22 (1997), no. 9-10, 1629-1646.

[12] Cianchi, A.: An optimal interpolation theorem of Marcinkiewicz type in Orlicz spaces. J. Funct. Anal. 153 (1998), no. 2, 357-381.

[13] Cianchi, A.: Hardy inequalites in Orlicz spaces. Trans. Amer. Math. Soc. 351 (1999), no 6, 2459-2478.

[14] Cianchi, A.: A fully anisotropic Sobolev inequality. Pacific J. Math. 196 (2000), no. 2, 283-295.

[15] Cwikel, M. and Pustylnik, E.: Sobolev type embeddings in the limiting case. J. Fourier Anal. Appl. 4 (1998), no. 4-5, 433-446.

[16] Donaldson, D. T. and Trudinger, N. S.: Orlicz-Sobolev spaces and embedding theorems. J. Functional Analysis 8 (1971), 52-75.

[17] Edmunds, D. E., Gurka, P. And Opic, B.: Double exponential integrability of convolution operators in generalized Lorentz-Zygmund spaces. Indiana Univ. Math. J. 44 (1995), no. 1, 19-43.

[18] Edmunds, D. E., Kerman, R. A. And Pick, L.: Optimal Sobolev imbeddings involving rearrangement invariant quasi-norms. J. Funct. Anal. 170 (2000), no. 2, 307-355.

[19] Fusco, N., Lions, P. L. And Sbordone, V.: Sobolev embedding theorems in borderline cases. Proc. Amer. Math. Soc. 124 (1996), no. 2, 561565 .

[20] Greco, L. and Moscariello, G.: An embedding theorem in LorentzZygmund spaces. Potential Anal. 5 (1996), no. 6, 581-590.

[21] Hansson, K.: Imbedding theorems of Sobolev type in potential theory. Math. Scand. 45 (1979), no. 1, 77-102. 
[22] Klimov, V.S.: Imbedding theorems and geometric inequalities. Izv. Akad. Nauk SSSR Ser. Mat. 40 (1976), no. 3, 645-671. Translated in Math. USSRIzv. 10 (1976), 615-638.

[23] KolyadA, V.I.: On the differential properties of the rearrangements of functions. In Progress in Approximation Theory (Tampa, FL, 1990), 333352. Springer Ser. Comput. Math. 19. Springer, New York, 1992.

[24] MaZ'YA, V. M.: Sobolev spaces. Springer Series in Soviet Mathematics. Springer-Verlag, Berlin, 1985.

[25] O'NeIL, R.: Convolution operators in $L(p, q)$ spaces. Duke Math. J. 30 (1963), 129-142.

[26] Peetre, J.: Espaces d' interpolation et théorème de Soboleff. Ann. Inst. Fourier 16 (1966), 279-317.

[27] Pohozhaev, S. I.: On the imbedding Sobolev theorem for $p l=n$. Doklady Conference, Section Math. Moscow Power Inst. (1965), 158-170 (Russian).

[28] TARTAR, L.: Imbedding theorems of Sobolev spaces into Lorentz spaces. Boll. Unione Mat. Ital. Sez. B Artic. Ric. Mat. (8) 1 (1998), no. 3, 479-500.

[29] Talenti, G.: An embedding theorem. In Partial differential equations and the calculus of variations, Vol. II, 919-924. Progr. Nonlinear Differential Equations Appl. 2. Birkhäuser, Boston, 1989.

[30] Troisi, M.: Teoremi di inclusione per spazi di Sobolev non isotropi. Ricerche Mat. 18 (1969), 3-24.

[31] Trudinger, N.S.: On imbeddings into Orlicz spaces and some applications. J. Math. Mech. 17 (1967), 473-483.

[32] Trudinger, N.S.: An imbedding theorem for $H^{0}(G, \Omega)$ spaces. Studia Math. 50 (1974), 17-30.

[33] Yudovich, V.I.: Some estimates connected with integral operators and with solutions of elliptic equations. Dokl. Akad. Nauk SSSR 138 (1961) 805-808. Translated in Soviet Math. Dokl. 2 (1961), 746-749.

[34] Ziemer, W. P.: Weakly differentiable functions. Graduate Texts in Mathematics 120. Springer-Verlag, New York, 1989.

Recibido: 6 de junio de 2002

Andrea Cianchi Dipartimento di Matematica e Applicazioni per l'Architettura

Università di Firenze Piazza Ghiberti 27 50122 Firenze, Italy cianchi@unifi.it 